\title{
DESIGN FOR BIT ERROR RATE ESTIMATION OF HIGH SPEED SERIAL LINKS
}

\author{
A THESIS \\ Submitted to \\ the Temple University Graduate Board \\ in Partial Fulfillment \\ of the Requirements for the Degree \\ MASTER OF SCIENCE IN ELECTRICAL ENGINEERING \\ by \\ Ujjwal Guin \\ August, 2010
}

Thesis Committee:

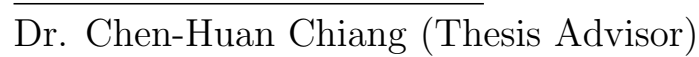

Dr. Saroj K. Biswas (Committee Member)

Dr. Li Bai (Committee Member) 


\begin{abstract}
High-speed serial links in modern communication systems often require the Bit-Error-Rate (BER) to be at the level of $10^{-12}$ or lower. From the industry perspective, major drawbacks in high volume production test for the serial links with low BER are the excessive test time for comparing each captured bit for error detection and costly instrumentation. In this thesis, we focus on developing a novel BER estimation methodology and its implementation. We propose a novel BER estimation methodology and an effective self-test system, which not only eliminates the usage of expensive measuring instruments, but also significantly reduces the test time.

In the proposed BER estimation, we show that the total jitter (TJ) spectral information of a test SerDes is successfully estimated from the known TJ distribution of a golden SerDes. We propose a novel BER estimation formula that incorporates not only the TJ spectral information of the serial data, but also the TJ spectral information of the recovered clock. Our proposed estimation formula enables efficient BER estimation without excessive test time, and its accuracy does not depend on the jitter present in the serial data stream of the SerDes. The experimental results demonstrate that the test time for the proposed BER estimation is in the order of seconds, which translates to the test time savings of more than hundred times compared to the traditional BER measurement for the same accuracy.

To implement the proposed BER estimation methodology, we have developed a novel time-to-digital converter (TDC). This design effectively measures the delay between two signals and converts it into the digital format. Performance of the TDC has been evaluated and presented using ModelSim and SPICE simulation.
\end{abstract}




\section{ACKNOWLEDGEMENTS}

The research could not be accomplished without the guidance and support of professors, friends and family members. First of all, I would like to thank my advisor, Dr. Chen-Huan Chiang. He always supports me and gives me great advices during this challenging period. I am grateful for his guidance and am privileged to work with him. I would also like to thank my committee members, Professor Li Bai and Professor Saroj Biswas, for serving my committee and technical guidance. Their dedication to research has inspired me and has significantly influenced my research.

I would like to express my gratitude towards my friends at Temple University. I cherish good memories in Philadelphia with them Dhiritiman, Satish, Nabeel, Avirup, and Debasish, to name just a few.

Finally, I give my warmest thanks to my parents. Their unconditional love and continual encouragement have been a great source of strength that allowed me to complete this work. I would like to dedicate this dissertation to my parents. 


\section{TABLE OF CONTENTS}

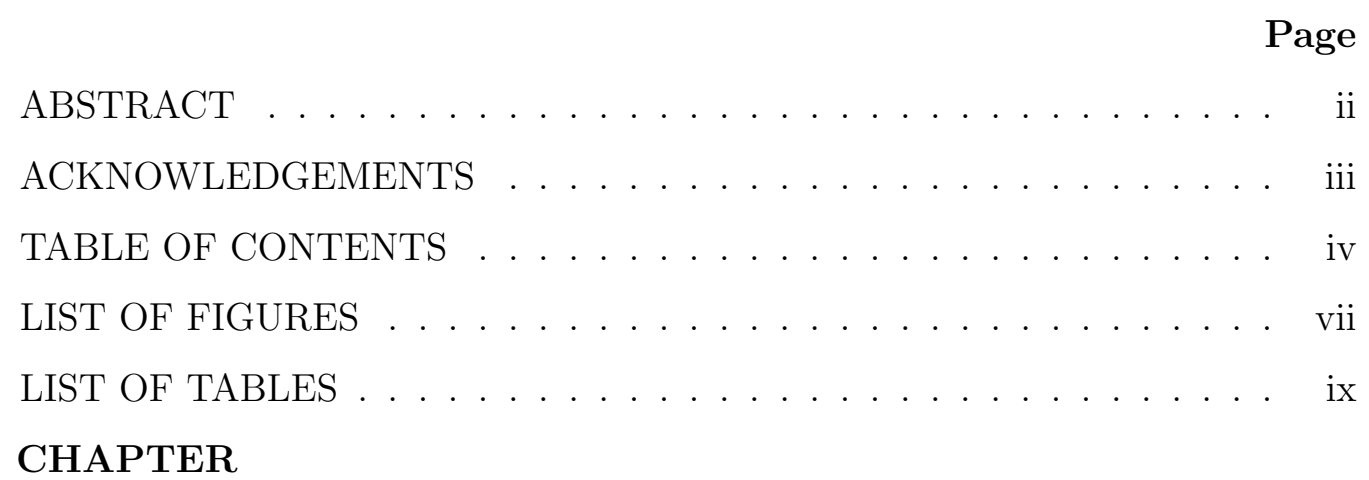

1 INTRODUCTION . . . . . . . . . . . . . . . . . . . . . 1

1.1 Introduction . . . . . . . . . . . . . . 1

1.2 Research Objectives . . . . . . . . . . . . . . . . 2

1.3 Thesis Outline . . . . . . . . . . . . . . . . 3

2 INTRODUCTION TO SERDES . . . . . . . . . . . . . . 4

2.1 Basic Structure of SerDes . . . . . . . . . . . . . . 4

2.1 .1 Transmitter ................... . . . 4

2.1.1.1 Coding Logic . . . . . . . . . . . . . 4

2.1.1.2 Serializer ................. 5

2.1.1.3 Reference Clock . . . . . . . . . . . . 5

2.1.1.4 PLL Multiplier . . . . . . . . . . . . 5

2.1.1.5 Line Driver . . . . . . . . . . . 6

2.1 .2 Receiver ................... 6

2.1.2.1 CDR Circuit . . . . . . . . . 6

2.1.2.2 Deserializer . . . . . . . . . . . . 7

2.1.2.3 Decoding Logic and Frame Alignment . . . . . 7

2.1.2.4 Line Receiver . . . . . . . . . . . . . . 7

2.2 Jitter . . . . . . . . . . . . . . . . . . . . 8 
2.2.1 Random Jitter . . . . . . . . . . . . . . . . . . 8

2.2 .2 Deterministic Jitter . . . . . . . . . . . . . . 8

2.2.3 Jitter Propagation in the Serial Communication system ........................... 10

2.3 Bit Error Rate . . . . . . . . . . . . . . . . . . . . 12

2.3.1 Measurement of BER using Direct Method . . . . . . . . . 13

2.3.2 Measurement of BER using dual-Dirac Method . . . . . . 13

3 PROPOSED MODEL FOR BER ESTIMATION . . . . . . . . . . . 17

3.1 Motivation . . . . . . . . . . . . . . . 17

3.2 Proposed BER Estimation . . . . . . . . . . . . . . . . 18

3.3 Proposed Design _. . . . . . . . . . . . . . . . . . . 22

3.3.1 Golden SerDes . . . . . . . . . . . . . . . . 22

3.3.2 Test SerDes . . . . . . . . . . . . . . . . . . . . . . 24

3.3.3 Loop-back . . . . . . . . . . . . . . . . . . . . . . . 24

3.3.4 Time-to-Digital Converter (TDC) . . . . . . . . . . . . 24

3.3.5 BER Estimator . . . . . . . . . . . . . . . . . . 24

3.4 Simulation Results . . . . . . . . . . . . . . . . . . 25

4 TIME-TO-DIGITAL CONVERTER . . . . . . . . . . . . . . . 30

4.1 Prior work of Time-to-Digital Converter . . . . . . . . . . . . . . 30

4.2 Proposed Time-to-Digital Converter . . . . . . . . . . . . . . 31

4.3 Simulation Results . . . . . . . . . . . . . . . . . . 34

4.4 Modifications in the Proposed Design . . . . . . . . . . . . . 36

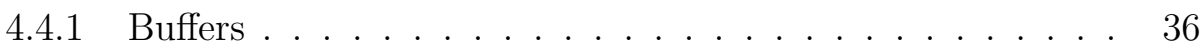

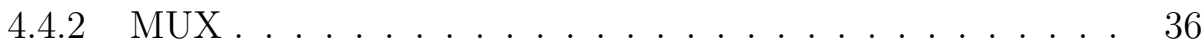

4.4 .3 D Flip-flops . . . . . . . . . . . . . . . . . . . 40

4.4.4 Final Design and Simulation . . . . . . . . . . . . . . 42

4.5 Application: Jitter Measurement . . . . . . . . . . . . . . 44

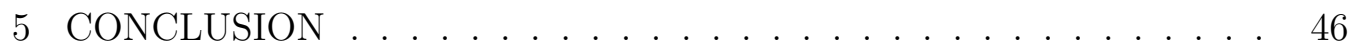


5.1 Contributions . . . . . . . . . . . . . . . . . . 46

5.2 Conclusion . . . . . . . . . . . . . . . . . 47

5.3 Future Work . . . . . . . . . . . . . . . . . . . . . . . . . 48

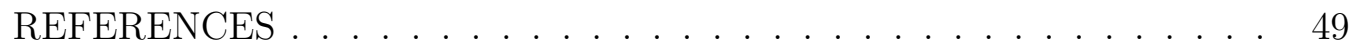
APPENDIX

A MATLAB CODES FOR THE TJ SPECTRAL INFORMATION ESTI-

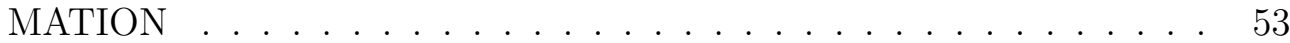

B TSPICE CODE FOR THE PROPOSED TIME-TO-DIGITAL CON-

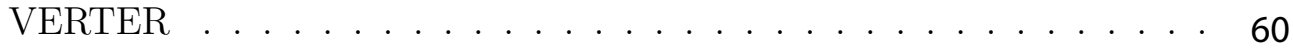




\section{LIST OF FIGURES}

2.1 Block diagram of the Transmitter $[3] \ldots \ldots$. . . . . . . . 5

2.2 Block diagram of the Receiver $[3] \ldots \ldots 6$

2.3 CDR Architecture $[13] \ldots \ldots \ldots$. . . . . . . . . . . 7

2.4 The random jitter with its density function $[2] \ldots \ldots$

2.5 The deterministic jitter with its density function $[2] \ldots 9$

2.6 Jitter family tree $[2] \ldots \ldots$. . . . . . . . . . . . . . . 10

2.7 The characteristics of total jitter $[2] \ldots \ldots \ldots 11$

2.8 Types of jitter generated in the various stages of the serial communication system $[2] \ldots \ldots \ldots \ldots$

2.9 The direct measurement of BER $[14] \ldots \ldots$. . . . . . . . . . 13

2.10 The convolution of sum of two delta functions separated by DJ and a Gaussian RJ distribution with variance $\sigma[11] \ldots \ldots$

2.11 Characteristic of a CDR circuit $[12] \ldots \ldots . \ldots 16$

3.1 Data and recovered clock waveforms with TJ Distribution . . . 20

3.2 The proposed design for BER estimation . . . . . . . . . . . 23

3.3 a) The TJ distribution of the Golden SerDes b) The TJ distribution of the Test SerDes c)The resultant TJ distribution . . . . . . 26

3.4 Estimation of mean and variance . . . . . . . . . . . . . . 28

4.1 Time-to-Digital Converter . . . . . . . . . . . . . . . . . 32

4.2 Modelsim simulation waveform of the proposed Time-to-Digital Converter .......................... 35

4.3 Modelsim simulation waveform of the proposed Time-to-Digital

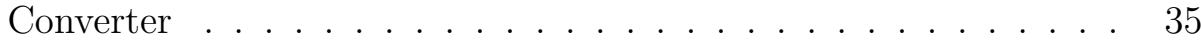


4.4 The TSPICE simulation for the buffers used in this design . . . . 37

4.5 TSPICE simulation of MUX . . . . . . . . . . . . . . 38

4.6 Proposed Time-to-Digital Converter after MUX delay Compensation 39

4.7 TSPICE simulation of DFF . . . . . . . . . . . . . . 40

4.8 Proposed Time-to-Digital Converter after clock-to- $\bar{Q}$ delay Com-

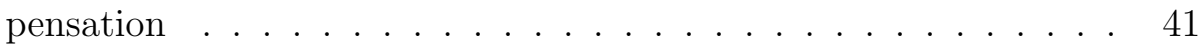

4.9 The complete proposed Time-to-Digital Converter . . . . . . . . . 42

4.10 The TSPICE simulation for the proposed Time-to-Digital Converter 43

4.11 Set-up for the jitter measurement of a system . . . . . . . . . . . 44

4.12 Jitter measurement using our proposed Time-to-Digital Converter 45 


\section{List of Tables}

3.1 Estimation of $\mu_{R} \ldots \ldots \ldots \ldots \ldots \ldots$

3.2 Estimation of $\mu_{L} \ldots \ldots \ldots \ldots \ldots$

3.3 Estimation of $\sigma \ldots \ldots \ldots \ldots \ldots \ldots \ldots$ 


\section{CHAPTER 1}

\section{INTRODUCTION}

\subsection{Introduction}

The data transfer rate in the modern systems increases significantly during past 15 years. The shifting of data transfer from parallel to serial makes this possible to increase. Today, 10-40 Gbps (giga bits per sec) systems are available for the Gigabit Ethernet and SONET that were one Gbps in 1995. In the computer area, I/O buses are available at 2.5-3 Gbps for the PCI express and serial ATA respectively that were $100 \mathrm{Mbps}$ in 1995 . Moreover, up to $4.8 \mathrm{Gbps} \mathrm{I} / \mathrm{O}$ buses are available for PCI Express generation II and fully buffered DIMM (Dual In-line Memory Module). These increased data rates result high-speed applications. It is assumed that this trend will continue for another decade or so. The increased data rate creates new opportunities for the technology development. At first, it was assumed that a serial link must be inferior to a parallel one, because it can transmit less data on each clock tick. However, with the advancement of the technology, it has verified that a serial links can be clocked considerably faster than parallel links, and achieve a higher data rate. A number of factors allow a serial link to be clocked at much faster rate. First, the clock skew between different channels is not an issue because the clock is embedded in the data and transmitted asynchronously (self-synchronous links). Secondly, a serial connection requires less interconnection and hence occupies less space that results better isolation of the channel from its surroundings. Finally, crosstalk is merely an issue because of fewer conductors are in proximity. However, there are always scopes 
for the further advancement. The data rate in the serial communication cannot increase significantly ever. The main source of this difficulty is that the available bit period (unit interval, UI) becomes smaller and smaller as data rate increases. The timing specifications must shrink as the proper portion to achieve the same performance of the BER which considers as the key factor of the quality of a high-speed serial link.

\section{$1.2 \quad$ Research Objectives}

The backbone of the serial architecture is the serializers and de-serializers (SerDes). They operate in the range of giga-bits per second (Gbps). The BER specification of a high quality SerDes is extremely small and is usually in the order of $10^{-12}$ or smaller. The test time to acquire such a small BER, is very high and is usually in terms of hours. It is because we need to compare each captured bit for error detection for as long as the time taken to capture at least 10 errors, i.e., $10 * 10^{12}$ bits of data.

Most of the oscilloscopes or measuring instruments measure total jitter at a given BER of $10^{-12}$ using bathtub curves by integrating the extrapolated total jitter (TJ) probability density function (pdf). Typical test times are approximately 20 minutes at $10 \mathrm{Gbit} / \mathrm{s}$, and a little more than one hour at $2.5 \mathrm{Gbit} / \mathrm{s}$, for a total jitter measurement that was done with a confidence level of better than $90 \%$ [13] at the $10^{-12}$ BER level. It is a significant improvement for the BER estimation, compared to the traditional bit-by-bit comparison for calculating BER. However, it is still far from a solution to high volume testing of the SerDes.

The first part of this research addresses the extraction of the TJ spectral information of the transmitted data of a test SerDes with the help of a golden SerDes whose TJ spectral information is known and previously measured by the conventional methods. The prime difficulty to implement this design is the delay 
measurement between the two signals. In Gbps range the UI is several hundred picoseconds. Due to the jitter, the UI varies by a small amount and is the order of picoseconds. It is practically impossible to measure such a small variation using conventional method. The later part of this research addresses a novel time-to-digital converter to measure such a small amount of delay.

\subsection{Thesis Outline}

The rest of this research proposal is organized as follows: Chapter 2 is an introduction of SerDes. It first describes the basic structures of the SerDes. Then various types of jitters in the serial communication system are introduced. At the end, we review the prior works regarding two popular methods of BER estimation. In Chapter 3, a proposed BER estimation method is presented. The design for implementing our proposed BER estimation method is also proposed. In Chapter 4, a proposed model for time-to-digital converter is presented. The future work is described in Chapter 5, along with the conclusion of the thesis. 


\section{CHAPTER 2}

\section{INTRODUCTION TO SERDES}

\subsection{Basic Structure of SerDes}

The SerDes or multi-gigabit transceiver [1]- [3] basically consists of separate transmitters and receivers for the input and output respectively. These blocks convert data between serial data and parallel data in each direction.

\subsubsection{Transmitter}

The transmitter accepts parallel data and converts it into a serial format. It consists of a coding logic, a serializer, a PLL frequency multiplier, a reference clock source and a line driver. The reference clock is the ultimate source of system timing. To define the timing of logic transitions, the transmitter multiplies the reference clock by an appropriate factor to get the desired data transmission rate. The block diagram of the transmitter is shown in the Figure 2.1.

\subsubsection{Coding Logic}

Coding logic consists of Transmit FIFO (First In First Out) and Line Decoder. Transmit FIFO allows for the storing of the incoming data before transmission. Line decoder encodes the data into a more line friendly format. It removes a long sequence of zeroes and ones and it adjusts the proper number of zeroes and ones in the data stream. $8 \mathrm{~b} / 10 \mathrm{~b}$ is the common line encoding scheme [1]. It is a value look up type encoding scheme where 8-bit words are transmitted into 10-bit symbols. 


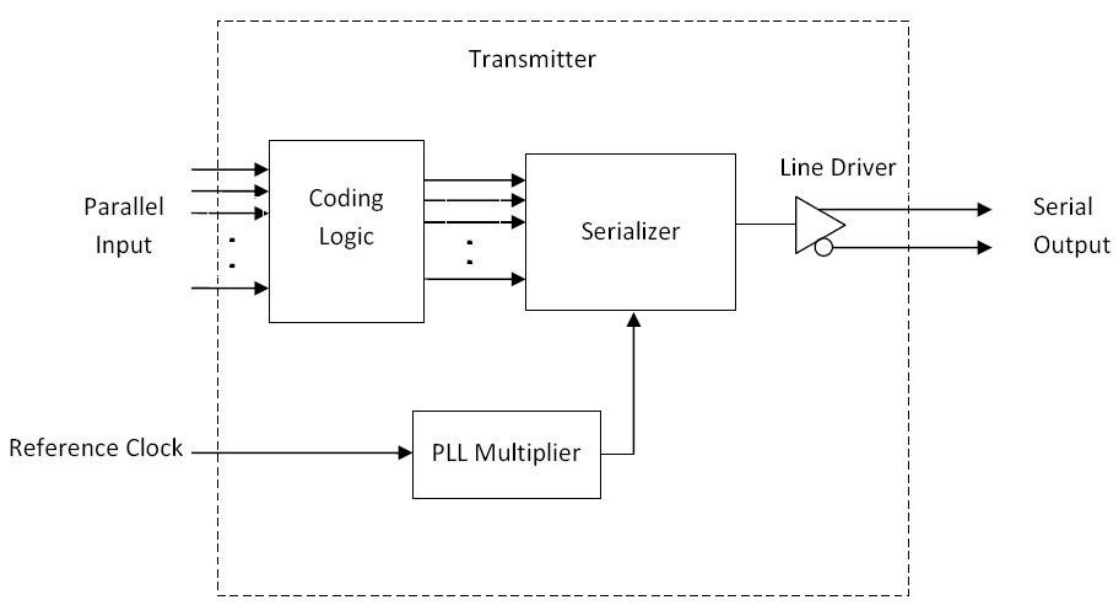

Figure 2.1: Block diagram of the Transmitter [3]

\subsubsection{Serializer}

It takes $n$ bits of parallel data changing at a rate of $y$ and converts this to serial stream with a rate of $n * y$.

\subsubsection{Reference Clock}

The requirement for the reference clock of a multi-gigabit transceiver has very tight specification. It includes a tight frequency requirement with PPM (parts per million) frequency error. Along with this, it will also have a strict jitter requirements defined in terms of unit intervals (UI). This clock is generated using the crystal oscillator.

\subsubsection{PLL Multiplier}

Typically, the reference clock oscillates at a rate much lower than the data rate. This unit multiplies the reference clock rate with appropriate number to obtain the desired data rate. 


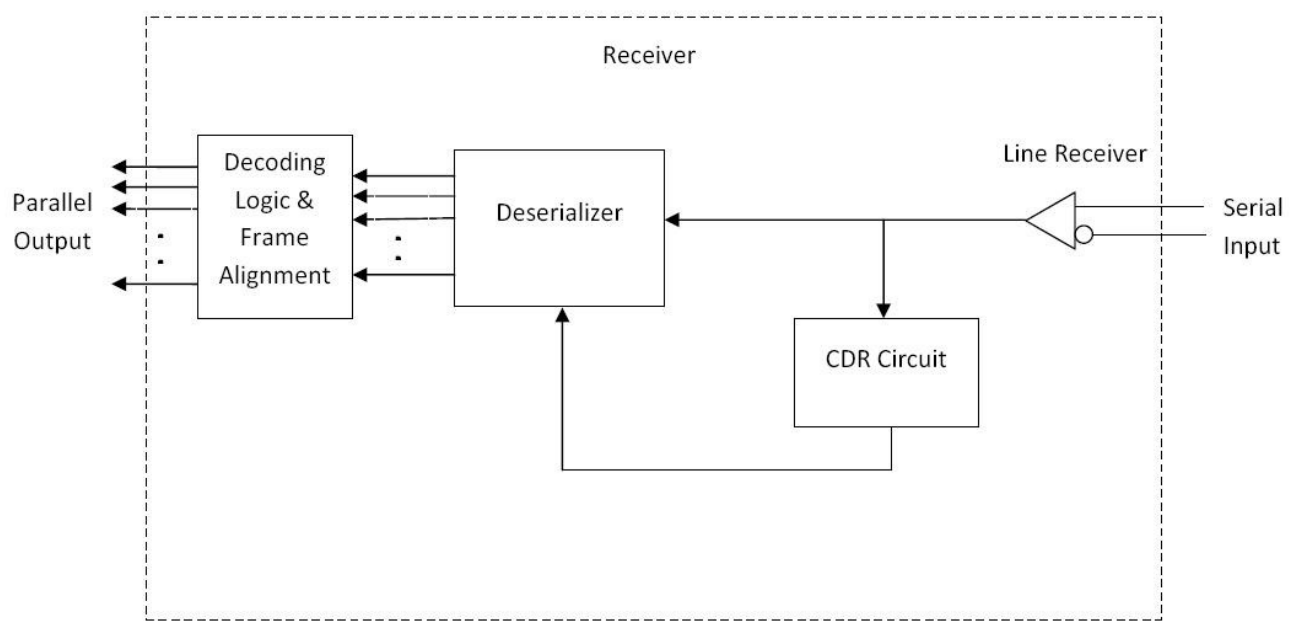

Figure 2.2: Block diagram of the Receiver [3]

\subsubsection{Line Driver}

Analog transmission circuit often allows varying drive strengths. It may also allow for pre-emphasis of transitions.

\subsubsection{Receiver}

The receiver accepts the high-speed serial data. It consists of a line receiver, a clock and data recovery (CDR) circuit, a deserializer and a decoding logic and frame alignment. The internal CDR circuit recovers the clock from the data stream. The deserializer converts this serial data to its parallel counterpart. The block diagram of the receiver is shown in the Figure 2.2

\subsubsection{CDR Circuit}

CDR circuit is the backbone of the receiver of the multi-gigabit transceiver. In serial communication, clock and data are transmitted simultaneously. CDR circuit recovers the clock from the data stream. It incorporates bang-bang (binary) 


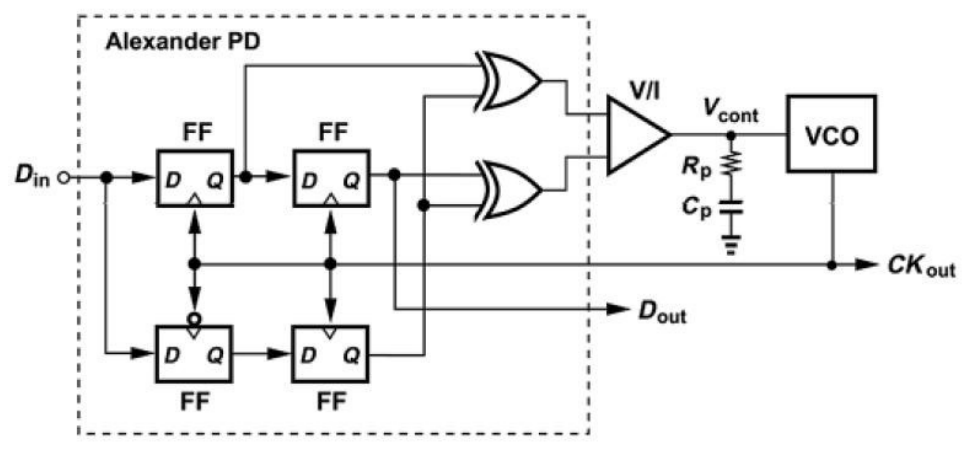

Figure 2.3: CDR Architecture [13]

phase detector, charge pump circuit and voltage controlled oscillator (VCO). The CDR architecture is shown in the Figure 2.3

\subsubsection{Deserializer}

It takes $n$ bits of serial data changing at a rate of $y * n$ and converts this to parallel data with a rate of $y$.

\subsubsection{Decoding Logic and Frame Alignment}

Line decoder decodes the line encoded data to plain data. The word boundary detection circuitry detects the comma character [1] and aligns the frames.

\subsubsection{Line Receiver}

Analog receive circuitry includes a differential receiver and may include active or passive equalization. 


\subsection{Jitter}

The definition of jitter in [6] is " the short term phase variation of the significant instants of a digital signal from their ideal positions in time". Jitter causes non cumulative phase variation in the serial data. The jitter probability density function (pdf) gives the probability for a given logic transition to differ from the ideal position. In the analysis of serial data systems, jitter or total jitter (TJ) [2] is divided into two distinguished catagories, i.e., random jitter (RJ) and deterministic jitter (DJ).

\subsubsection{Random Jitter}

Random jitter $(\mathrm{RJ})$ is characterized by a Gaussian distribution or normal distribution. It is believed that RJ is generated from the thermal noise of the electric circuit, which does have a Gaussian distribution. Moreover, it is also generated because of a huge number of processes with small magnitudes. The central limit theorem states that composite effect of many uncorrelated noise sources, regardless of the distributions, approaches a Gaussian distribution. Figure 2.5 shows the Random Jitter with its density function,

Since the RJ is described by the normal distribution, it will be completely characterized by the mean $(\mu)$ and the standard deviation $(\sigma)$. RJ is unbounded that means it does not have well defined peak to peak value. The PDF of the RJ is

$$
R J(x)=\frac{1}{\sqrt{2 \pi} \sigma} \exp ^{-\frac{(x-\mu)^{2}}{2 \sigma^{2}}}
$$

\subsubsection{Deterministic Jitter}

Deterministic Jitter (DJ) is characterized by a small number of processes and they are not necessarily to be independent. The name deterministic suggests that it 


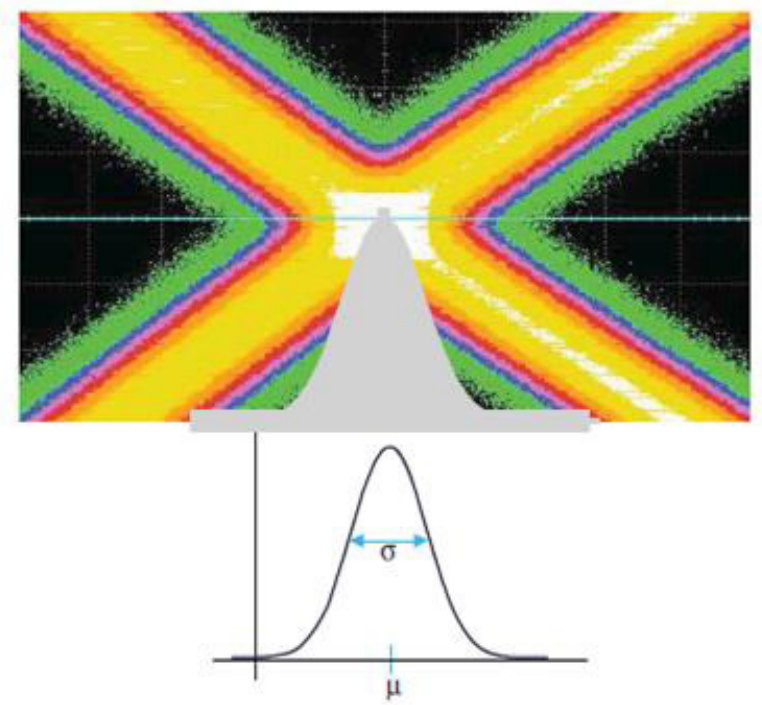

Figure 2.4: The random jitter with its density function [2]

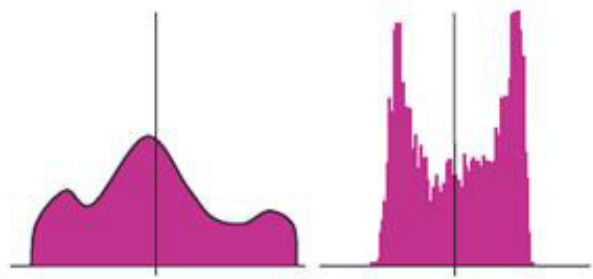

Figure 2.5: The deterministic jitter with its density function [2]

can be accurately predicted if the system is known completely. DJ is bounded and it has a definite peak-to-peak DJ (p-p) value. It is caused mainly due to electromagnetic interference (EMI), channel frequency response, reflections in the channel etc. Figure 2.5 shows the deterministic jitter with its density function,

The DJ is divided into three major categories, i.e., Periodic Jitter (PJ), Data Dependent Jitter (DDJ) and Bounded Uncorrelated Jitter(BUJ). The DDJ is further divided into two categories, i.e., Duty Cycle Distortion (DCD) and Inter Symbol Interference (ISI). The detailed descriptions can be found in [2]. The 


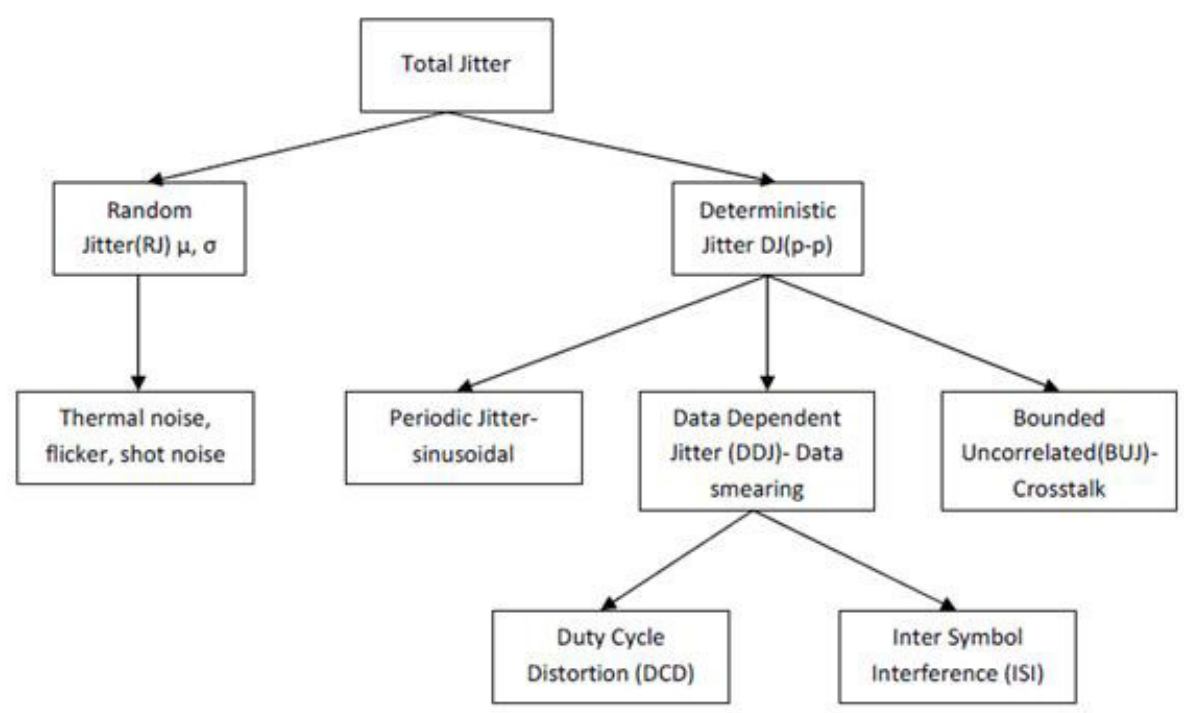

Figure 2.6: Jitter family tree [2]

Figure 2.6 describes the complete jitter tree.

The Figure 2.7 shows the total jitter (TJ) when RJ and DJ are present in the data.

\subsubsection{Jitter Propagation in the Serial Communication system}

Figure 2.8 shows how jitter is generated and propagated through a communication channel.

The Reference Clock: The reference clock circuit generates primarily RJ, PJ and DCD. The main source of RJ is the thermal noise of the oscillator. PJ is generated from the spurious sideband resonance of the oscillator and DCD is generated from the nonlinearities in the oscillator.

The Transmitter (TX): The transmitter adds RJ, ISI and PJ to the data 

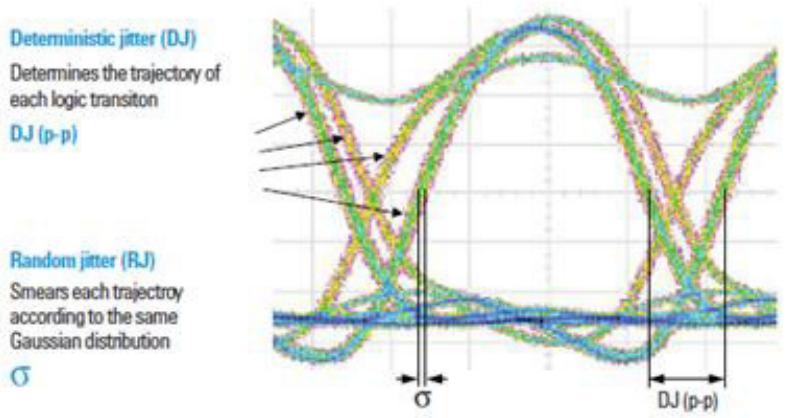

Figure 2.7: The characteristics of total jitter [2]

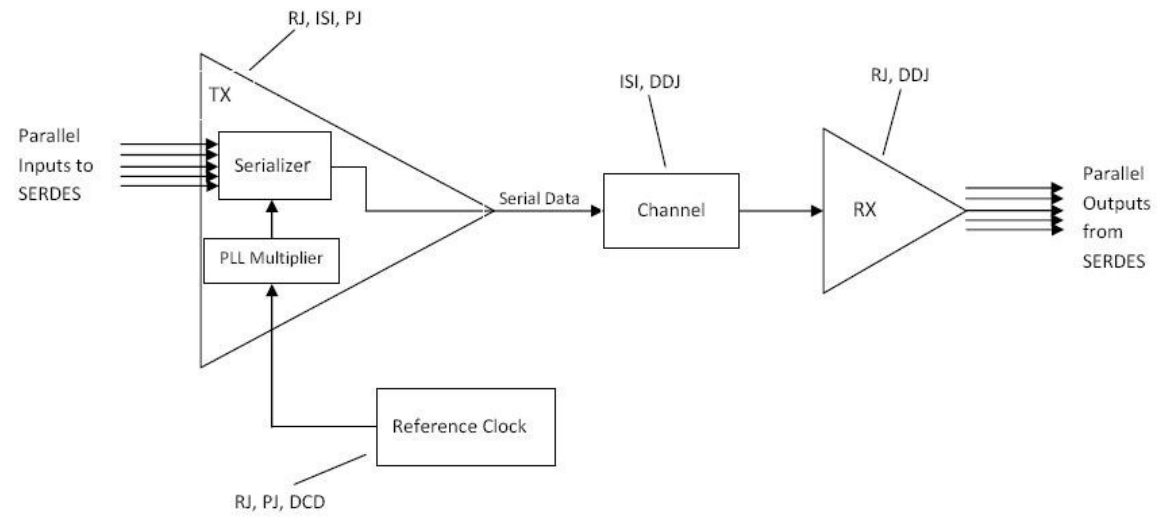

Figure 2.8:

Types of jitter generated in the various stages of the serial communication system $[2]$ 
stream. RJ is caused due to the thermal noise of the transmitter. ISI is generated from the frequency response of the internal transmission lines and PJ is due to EMI.

Again, the PLL multiplier adds its own RJ from the voltage controlled oscillator (VCO) of the PLL circuitry and also amplifies the jitter generated from the reference clock. The effect of frequency multiplication [4] by a factor $n$, multiplies the phase noise power to carrier ratio by $n^{2}$.

The Transmission Channel: The impedance mismatch of TX and transmission channel and the skin effect cause both attenuation and non-uniform frequency response that cause ISI [5]. The ISI introduced by the channel is affected by the DCD. The combination of ISI and DCD is called Data Dependent Jitter (DDJ). Hence,

$$
D D J=I S I+D C D
$$

and

$$
p d f(D D J)=p d f(I S I) * p d f(D C D)
$$

The Receiver (RX): RJ is generated in the receiver from the VCO of the CDR circuit and thermal effect of the circuit elements and DDJ is from the internal circuitry.

\subsection{Bit Error Rate}

Bit error rate (BER) is defined as the ratio of the number of errors $\left(N_{\text {err }}\right)$ to the total bits $(\mathrm{N})$ transmitted.

$$
B E R=\frac{N_{e r r}}{N}
$$




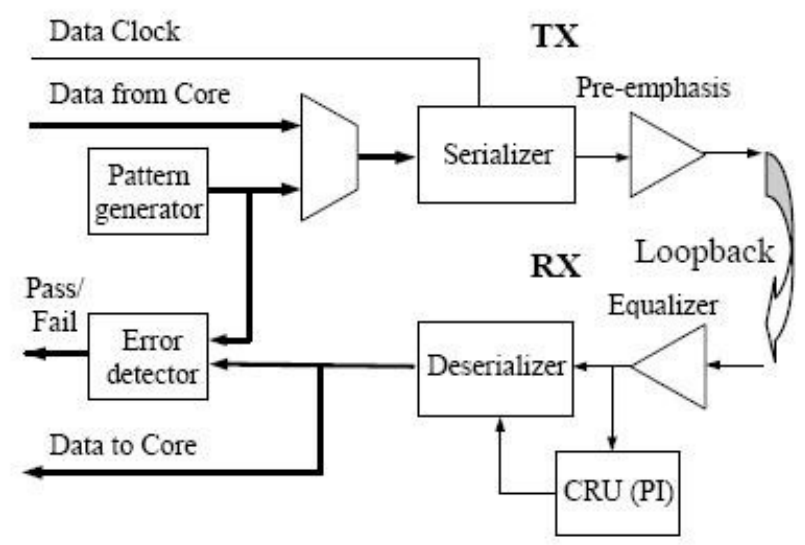

Figure 2.9: The direct measurement of BER [14]

\subsubsection{Measurement of BER using Direct Method}

The direct method of BER measurement is shown in Figure 2.9. A simple pattern generator and an error detector are incorporated in the measurement. The SerDes has a phase interpolator (PI) in the clock recovery unit (CRU), which can introduce a fine resolution phase offset between the clock and the data. Thus, bit-error-rate (BER) measurements can be made at the center of the data eye as the capturing of data in the center of the data eye gives most accurate result. The BER would be calculated using the Equation 2.2. This method is regarded as the on-chip version of the Bit Error Rate Tester (BERT) [14]. The excessive test time for comparing each captured bit for error detection is the major bottleneck of this type of BER measurement.

\subsubsection{Measurement of BER using dual-Dirac Method}

The dual-Dirac model [11] is a tool for quickly estimating the spectral information of total jitter present in the serial data stream. The following assumptions are made during the estimation process. 
- RJ and DJ are independent

- RJ follows a Gaussian distribution and are completely described in terms of mean $(\mu)$ and variance $\left(\sigma^{2}\right)$.

- DJ follows a finite, bounded distribution.

- No jitter is present in the clock

The jitter distribution, that is the histogram of the crossing-point of an eye diagram, could be imagined as having three distinct regions as shown in Figure 2.10. First, in Region 1 (R1), the distribution is dominated by DJ at the crossing-point. Secondly, in Region 2 (R2), at time-delays farther from the crossing-point the distribution is increasingly dominated by RJ. Finally, in Region 3 (R3), far from the crossing point the tails follow the Gaussian RJ distribution. The asymptotic tails of the distribution usually cause errors at the level of $B E R<10^{-8}$. The ideal way to determine the behavior of the tails, and hence, TJ would be the convolution of RJ and DJ.

$$
\begin{array}{r}
T J(x)=R J(x) * D J(x) \\
B E R(x)=\rho_{T} \int_{-\infty}^{\infty} T J(x) \mathrm{d} x
\end{array}
$$

The detailed derivation of BER would be found at [11] and is

$$
B E R=2 * \rho_{T} * Q\left(\frac{T / 2-\mu_{L}}{\sigma}\right)
$$

where, $\quad Q(x)=\frac{1}{\sqrt{2 \pi}} \int_{x}^{\infty} \exp ^{-\frac{x^{2}}{2}} \mathrm{~d} x$

The dual-Dirac model does not consider the clock recovery. The CDR circuit can track the low frequency jitter. As we know, because of unbounded nature of RJ, CDR cannot track it. However, the CDR circuit can track the DJ completely 


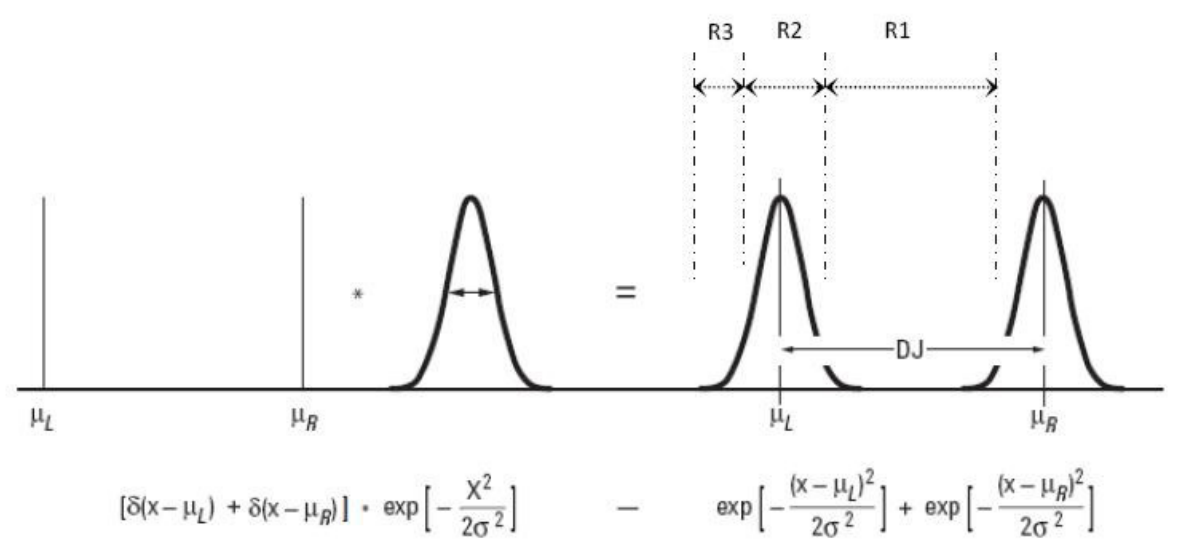

Figure 2.10:

The convolution of sum of two delta functions separated by DJ and a Gaussian RJ distribution with variance $\sigma$ [11]

or partially depending on its jitter transfer function. In that case, the above Equation 2.5 would no longer be valid. The modified formula [12] is

$$
B E R=2 * \rho_{T} * Q\left(\frac{T / 2-A_{e f f}}{\sigma_{e f f}}\right)
$$

where, $A_{\text {eff }}$ and $\sigma_{\text {eff }}$ would be different in the different regions of transfer characteristics of the CDR circuit that is shown in Figure 2.11.

The major drawback of this estimation process is that to estimate the BER, the prior knowledge of the DJ is required. Without it, it is impossible to estimate BER using the equation 2.6 


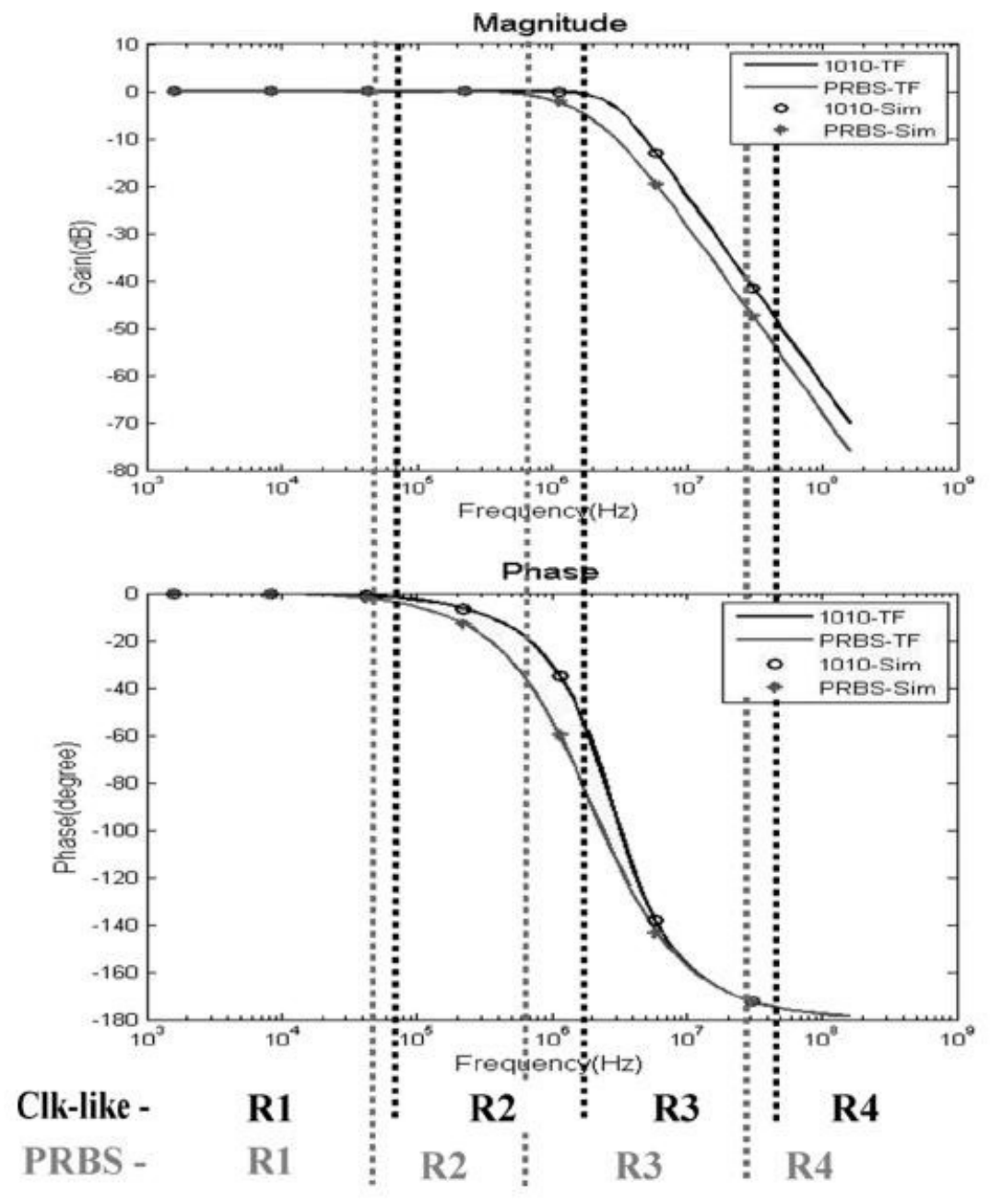

Figure 2.11: Characteristic of a CDR circuit [12] 


\section{CHAPTER 3}

\section{PROPOSED MODEL FOR BER ESTIMATION}

\subsection{Motivation}

As described in Section 2.3.2, the major drawback to estimate the BER using dual-Dirac method is to require the prior knowledge of DJ present in the data. In

addition, it requires an expensive measurement instrument, such as, high-speed oscilloscope or bit error rate tester (BERT). Furthermore, this method is not automated. It is highly tedious to get the bathtub plot using the oscilloscope or BERT. It is practically impossible to measure the BER of every SerDes manufactured in high volume. Thus, there is an urgent need for a test methodology to test the SerDeses efficiently for high volume manufacturing.

In the following sections, we will introduce a novel BER estimation model by considering the spectral information of the recovered clock. The spectral information of the TJ present on the data of a SerDes under test will be estimated using the spectral information of a golden SerDes. To implement the proposed BER estimation model, we propose a self-test system for high volume test. Finally, the MATLAB simulation for the estimation of the spectral information will be presented. 


\subsection{Proposed BER Estimation}

The key objective of this research is to reduce the test time for the BER estimation. The direct method for BER estimation not only requires a BER tester, but also also takes very long testing time. On the other hand, although the dual-Dirac model reduces the testing time but not suitable for the high volume testing.

For BER estimation, the variance $\left(\sigma^{2}\right)$ and mean $(\mu)$ of the TJ distribution are the parameters that must be estimated accurately. in our proposed test methodology, in order to automate the testing procedure, the spectral information, i.e., $\sigma$ and $\mu$, of a test SerDes will be accurately estimated with the help of a golden SerDes.

In our proposed procedure, there are three major assumptions made for the estimation of the spectral information of TJ of the test and golden SerDeses and they are,

- The TJ distribution of the golden SerDes and test SerDes are independent due to the process variation.

- The spectral information of the TJ distribution of the golden SerDes is measured previously with the help of a conventional testing method.

- TJ is a stationary phenomenon, i.e., a measurement of the spectral information on a given system, taken over an appropriate interval, will give the same result regardless of what time interval is initiated.

The total jitter is a random variable. If we add two independent random vari-

ables, their density functions will be convolved and the resultant distribution will be spread. In this research, we have selected the subtraction of the two total jitter random variables from the golden and the SerDes under test to generate 
the resultant TJ distribution. The SerDes under test will be referred as "test SerDes" in following sections. We have used Expectation Maximization (EM) algorithm [27], [28] and [29] to estimate the mean $(\mu)$ and variance $\left(\sigma^{2}\right)$ from the resultant TJ distribution. The notations for the variance and mean of the of the TJ distribution of the golden SerDes, test SerDes and the resultant are described in the table below,

\begin{tabular}{|c|c|c|}
\hline Parameter & : & Description \\
\hline$\sigma_{d, g}^{2}$ & & $\begin{array}{l}\text { Variance of the RJ component present in the serializer out- } \\
\text { put of Golden SerDes }\end{array}$ \\
\hline$\sigma_{c, g}^{2}$ & : & $\begin{array}{l}\text { Variance of the RJ component present in the recovered } \\
\text { clock of Golden SerDes }\end{array}$ \\
\hline$\mu_{R d, g}-\mu_{L d, g}$ & $:$ & DJ component in the serializer output of Golden SerDes \\
\hline$\mu_{R c, g}-\mu_{L c, g}$ & : & $\begin{array}{l}\text { DJ component in the recovered clock output of Golden } \\
\text { SerDes }\end{array}$ \\
\hline$\sigma_{d, t}^{2}$ & : & $\begin{array}{l}\text { Variance of the RJ component present in the serializer out- } \\
\text { put of Test SerDes }\end{array}$ \\
\hline$\sigma_{c, t}^{2}$ & : & $\begin{array}{l}\text { Variance of the RJ component present in the recovered } \\
\text { clock of Test SerDes }\end{array}$ \\
\hline$\mu_{R d, t}-\mu_{L d, t}$ & : & DJ component in the serializer output of Test SerDes \\
\hline$\mu_{R c, t}-\mu_{L c, t}$ & : & DJ component in the recovered clock output of Test SerDes \\
\hline$\sigma_{d, r}^{2}$ & : & $\begin{array}{l}\text { Variance of the RJ component present in the resultant data } \\
\text { jitter }\end{array}$ \\
\hline$\sigma_{c, r}^{2}$ & : & $\begin{array}{l}\text { Variance of the RJ component present in the resultant } \\
\text { clock jitter }\end{array}$ \\
\hline$\mu_{R d, r}-\mu_{L d, r}$ & $:$ & DJ component in the resultant data jitter \\
\hline$\mu_{R c, r}-\mu_{L c, r}$ & & DJ component in the resultant clock jitter \\
\hline
\end{tabular}




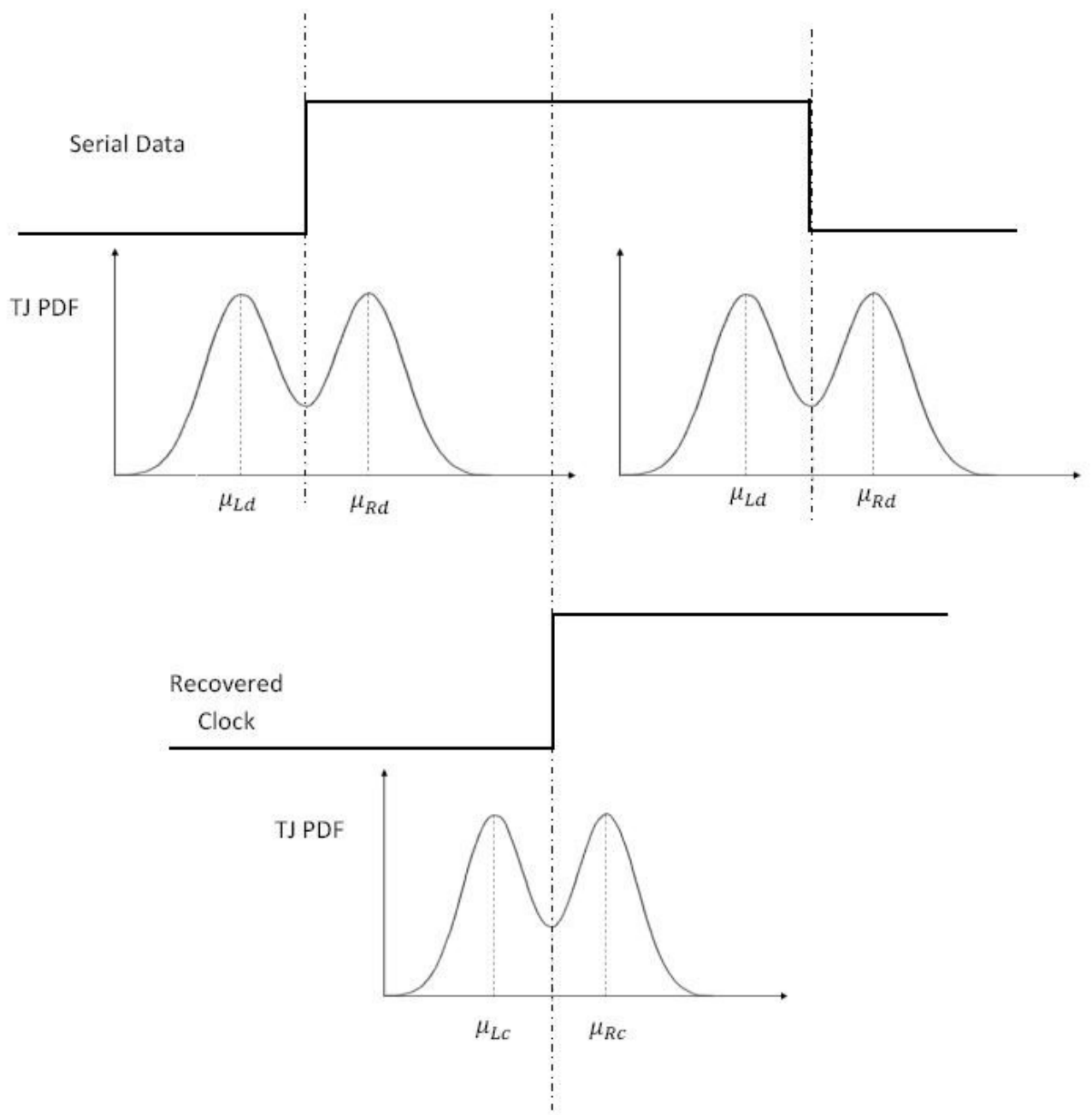

Figure 3.1: Data and recovered clock waveforms with TJ Distribution 
The relationship of the mean and variances of the above three distributions are

$$
\begin{gathered}
\sigma_{d, r}^{2}=\sigma_{d, g}^{2}+\sigma_{d, t}^{2} \\
\sigma_{c, r}^{2}=\sigma_{c, g}^{2}+\sigma_{c, t}^{2} \\
\mu_{L d, r}=\mu_{L d, g}+\mu_{L d, t} \\
\mu_{L c, r}=\mu_{L c, g}+\mu_{L c, t}
\end{gathered}
$$

Unlike previously proposed test method in [12] using dual-Dirac method, both the spectral information of the TJ present on the data and the recovered clock are considered for BER estimation in our proposed methodology. Figure 3.1 shows the data and recovered clock waveform with their TJ distribution. In [12], as the clock varies from its ideal sampling position due to the jitter transfer characteristics of the CDR and random jitter generated in the CDR circuit, the formula for the estimation of BER would be different in the different regions of the jitter transfer function of the CDR circuit. To develop a common formula for the estimation of BER in different regions of the CDR circuit, the TJ present in the recovered clock is incorporated in our BER estimation. As a result, the BER would be independent of the regions of the jitter transfer characteristic of the CDR circuit and can simply be estimated by the following equation,

$$
B E R=2 * \rho_{T} * Q\left(\frac{T / 2-A_{\text {eff }}}{\sigma_{\text {eff }}}\right)
$$

where,

$$
\begin{aligned}
& \sigma_{e f f}=\sqrt{\sigma_{d, t}^{2}+\sigma_{c, t}^{2}} \\
& A_{e f f}=\mu_{L d, t}-\mu_{L c, t}
\end{aligned}
$$




\subsection{Proposed Design}

The proposed self-test system for automatic BER estimation is described in Figure 3.2. It comprises of two SerDeses. One is the golden SerDes whose spectral information, variance $\left(\sigma^{2}\right)$ and mean $(\mu)$, are previously measured by the conventional method using oscilloscope or BERT. The main idea of the proposed test methodology is to estimate correctly the spectral information of the test SerDes with the help of the golden one. The common data is fed to both the SerDeses. The length of the paths from the data to the SerDeses must be equal to eliminate the delay difference. The serial output of the two transmitters are fed to a time-to-digital converter. The length of the paths must be of equal as well. To obtain the recovered clocks, the serial output of the transmitter is looped back to the receiver of the same SerDes. The recovered clock of each receiver is fed to a second time-to-digital converter. The output of the time-to-digital converter will be the phase differences of the two input signals in binary format. The detailed description will be introduced in Chapter 4. The histogram of the output of the time-to-digital converter will be resultant total jitter distribution.

In the following sections, the detailed descriptions of each units used in the proposed self-test system will be introduced.

\subsubsection{Golden SerDes}

The spectral information, $\sigma_{d}^{2}, \sigma_{c}^{2}, \mu_{L}, \mu_{R}$, of the serial data and recovered clock measured by the conventional method in advance. It is assumed that the spectral information would be constant during the testing. This SerDes is permanently placed in the test fixture. 


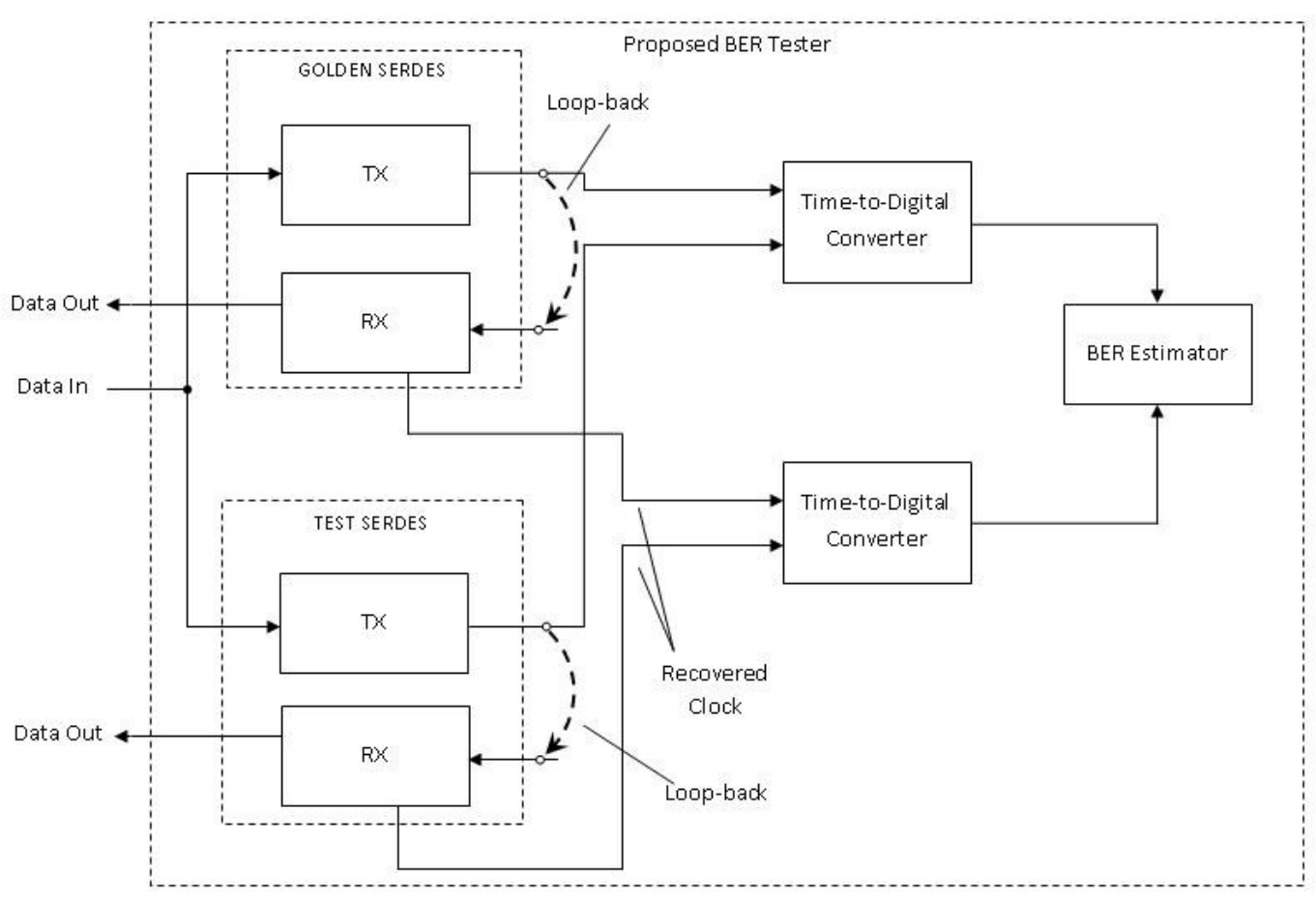

Figure 3.2: The proposed design for BER estimation 


\subsubsection{Test SerDes}

The spectral information, $\sigma_{d}^{2}, \sigma_{c}^{2}, \mu_{L}, \mu_{R}$, of the serial data and recovered clock are to be measured. This SerDes is not permanently fixed in the fixture and would be replaced after testing.

\subsubsection{Loop-back}

The output of the transmitter is fed to the receiver of the same SerDes to get the recovered clock from the receiver. This is very important as the spectral information of the recovered clock helps to estimate the BER with a simpler estimation method.

\subsubsection{Time-to-Digital Converter (TDC)}

This unit calculates the phase difference of the two signals and gives the binary output corresponding to the phase difference. We will describe this novel timeto-digital converter in Chapter 4.

\subsubsection{BER Estimator}

This unit takes the data from the TDC and makes the histogram with that data. Based on the histogram, it calculates all the spectral information such as $\sigma^{2}, \mu_{L}$ and $\mu_{R}$ using EM algorithm. This algorithm has been verified in MATLAB. However, We have not implemented this unit in hardware. Finally, the BER will be estimated using the complete set of spectral information of the test SerDes using the Equation 3.5. 


\subsection{Simulation Results}

A behavioral model of for the TDC with the EM algoorithm of the self-test system

has been simulated in MATLAB. However, we could not able to estimate the BER as the unavailability of the spectral information of TJ present on the recovered clock in the literature. The spectral information of the TJ present on the data of the golden SerDes is taken from a white paper of Agilent Technologies [11]. The $\mu_{L}, \mu_{R}$ and $\sigma$ of the golden SerDes are -13 ps, 13 ps and 5.7 ps respectively. We have assumed that the spectral information of the test SerDes are -14 ps, 14 ps and 5.5 ps respectively. We will estimate these parameters using our proposed method.

The TJ distribution of the golden SerDes and test SerDes and the resultant TJ distribution are generated in MATLAB and shown in the Figure 3.3. 

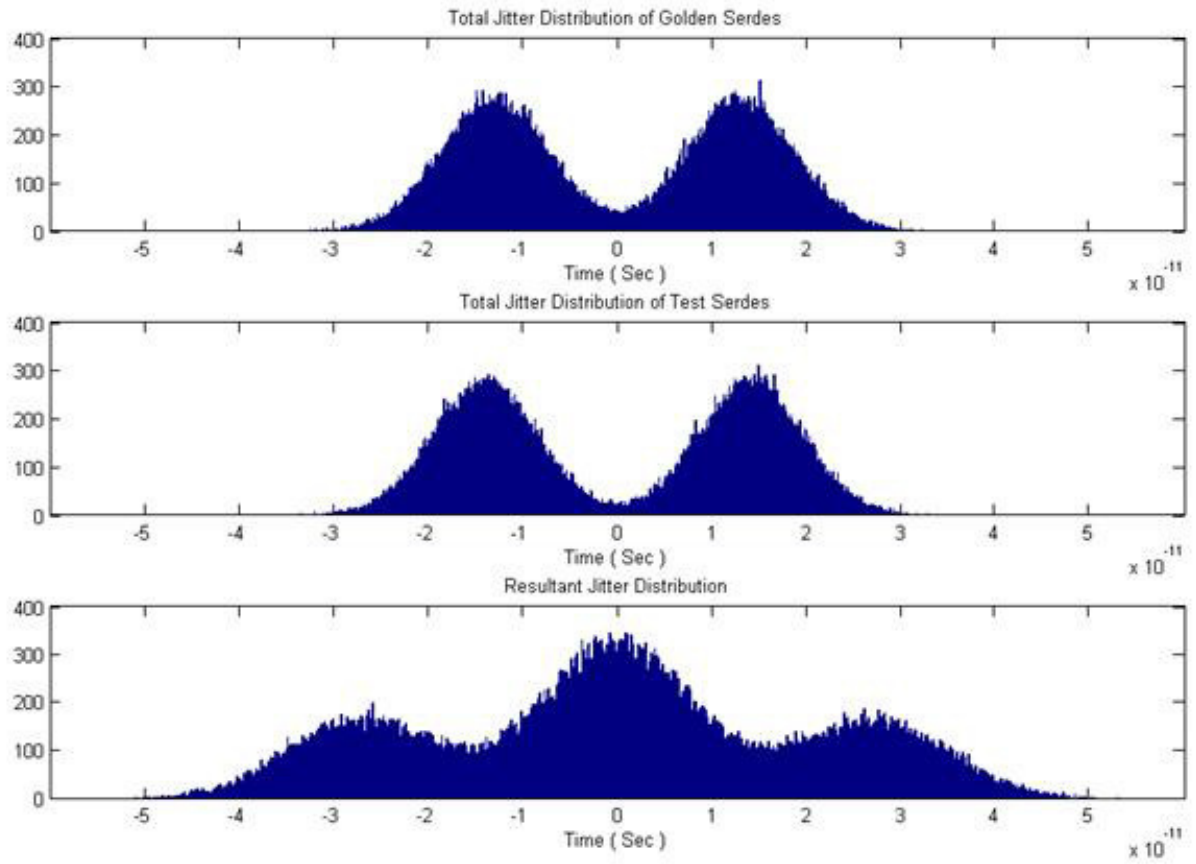

Figure 3.3:

a) The TJ distribution of the Golden SerDes b) The TJ distribution of the Test SerDes c)The resultant TJ distribution 
Table 3.1: Estimation of $\mu_{R}$

\begin{tabular}{|c|c|c|c|c|c|c|c|c|}
\hline $\begin{array}{c}\text { Measur- } \\
\text { ements }\end{array}$ & $10^{4}$ Bits & $\begin{array}{c}\% \\
\text { Error }\end{array}$ & $\begin{array}{c}2 * 10^{4} \\
\text { Bits }\end{array}$ & $\begin{array}{c}\% \\
\text { Error }\end{array}$ & $\begin{array}{c}5 * 10^{4} \\
\text { Bits }\end{array}$ & $\begin{array}{c}\% \\
\text { Error }\end{array}$ & $\begin{array}{c}10 * 10^{4} \\
\text { Bits }\end{array}$ & $\begin{array}{c}\% \\
\text { Error }\end{array}$ \\
\hline 1 & $14.30 \mathrm{ps}$ & 2.14 & $14.29 \mathrm{ps}$ & 2.07 & $13.90 \mathrm{ps}$ & 0.71 & $14.05 \mathrm{ps}$ & 0.36 \\
\hline 2 & $14.11 \mathrm{ps}$ & 0.79 & $13.96 \mathrm{ps}$ & 0.29 & $13.81 \mathrm{ps}$ & 1.36 & $13.88 \mathrm{ps}$ & 0.86 \\
\hline 3 & $13.61 \mathrm{ps}$ & 2.79 & $13.94 \mathrm{ps}$ & 0.43 & $14.01 \mathrm{ps}$ & 0.07 & $14.03 \mathrm{ps}$ & 0.21 \\
\hline 4 & $14.30 \mathrm{ps}$ & 2.14 & $14.29 \mathrm{ps}$ & 2.07 & $13.90 \mathrm{ps}$ & 0.71 & $13.93 \mathrm{ps}$ & 0.50 \\
\hline 5 & $14.28 \mathrm{ps}$ & 2.00 & $13.69 \mathrm{ps}$ & 2.21 & $14.05 \mathrm{ps}$ & 0.36 & $14.01 \mathrm{ps}$ & 0.07 \\
\hline 6 & $14.06 \mathrm{ps}$ & 0.43 & $14.00 \mathrm{ps}$ & 0.00 & $14.01 \mathrm{ps}$ & 0.07 & $13.92 \mathrm{ps}$ & 0.57 \\
\hline
\end{tabular}

We have run the simulation model several times to estimate $\mu$ and $\sigma$ using our proposed BER estimation methodology. Table 3.1, 3.2 and 3.3 summarize the results. We have run this proposed model to capture $10^{4}$ samples, $2 * 10^{4}$ samples, $5 * 10^{4}$ samples and $10^{5}$ samples to estimate the TJ spectral information. Table 3.1 represents the estimated $\mu_{R}$ values with different samples and time intervals. Based on the six measurements, the maximum variation of $\mu_{R}$ with respect to the actual value is $2.8 \%$ when the captured samples are $10^{4}$. However, the estimation accuracy increases significantly when larger number of samples are captured. The maximum variation is reduced to $0.86 \%$ when the captured samples are $10^{5}$. Table 3.2 and 3.3 summarize the estimation of $\mu_{L}$ and $\sigma$ of the TJ distribution of the test SerDes. The maximum variation of $\mu_{L}$ with respect to the actual value is $2.7 \%$ and $0.79 \%$ when the captured samples are $10^{4}$ and $10^{5}$ respectively and the maximum variation of $\sigma$ with respect to the actual value is $5.65 \%$ and $0.73 \%$ when the captured samples are $10^{4}$ and $10^{5}$ respectively. 
Table 3.2: Estimation of $\mu_{L}$

\begin{tabular}{|c|c|c|c|c|c|c|c|c|}
\hline $\begin{array}{c}\text { Measur- } \\
\text { ements }\end{array}$ & $10^{4}$ Bits & $\begin{array}{c}\% \\
\text { Error }\end{array}$ & $\begin{array}{c}2 * 10^{4} \\
\text { Bits }\end{array}$ & $\begin{array}{c}\% \\
\text { Error }\end{array}$ & $\begin{array}{c}5 * 10^{4} \\
\text { Bits }\end{array}$ & $\begin{array}{c}\% \\
\text { Error }\end{array}$ & $\begin{array}{c}10 * 10^{4} \\
\text { Bits }\end{array}$ & $\begin{array}{c}\% \\
\text { Error }\end{array}$ \\
\hline 1 & $-13.88 \mathrm{ps}$ & 0.86 & $-14.12 \mathrm{ps}$ & 0.86 & $-14.03 \mathrm{ps}$ & 0.21 & $-14.11 \mathrm{ps}$ & 0.79 \\
\hline 2 & $-14.32 \mathrm{ps}$ & 2.29 & $-14.21 \mathrm{ps}$ & 1.50 & $-14.00 \mathrm{ps}$ & 0.00 & $-13.94 \mathrm{ps}$ & 0.43 \\
\hline 3 & $-14.29 \mathrm{ps}$ & 2.07 & $-13.95 \mathrm{ps}$ & 0.36 & $-13.88 \mathrm{ps}$ & 0.86 & $-14.02 \mathrm{ps}$ & 0.14 \\
\hline 4 & $-13.88 \mathrm{ps}$ & 0.86 & $-14.12 \mathrm{ps}$ & 0.86 & $-14.00 \mathrm{ps}$ & 0.00 & $-13.90 \mathrm{ps}$ & 0.71 \\
\hline 5 & $-14.38 \mathrm{ps}$ & 2.71 & $-13.95 \mathrm{ps}$ & 0.36 & $-14.1 \mathrm{ps}$ & 0.71 & $-14.01 \mathrm{ps}$ & 0.07 \\
\hline 6 & $-13.81 \mathrm{ps}$ & 1.36 & $-14.10 \mathrm{ps}$ & 0.71 & $-14.15 \mathrm{ps}$ & 1.07 & $-13.90 \mathrm{ps}$ & 0.71 \\
\hline
\end{tabular}

Table 3.3: Estimation of $\sigma$

\begin{tabular}{|c|c|c|c|c|c|c|c|c|}
\hline $\begin{array}{c}\text { Measur- } \\
\text { ements }\end{array}$ & $10^{4}$ Bits & $\begin{array}{c}\% \\
\text { Error }\end{array}$ & $\begin{array}{c}2 * 10^{4} \\
\text { Bits }\end{array}$ & $\begin{array}{c}\% \\
\text { Error }\end{array}$ & $\begin{array}{c}5 * 10^{4} \\
\text { Bits }\end{array}$ & $\begin{array}{c}\% \\
\text { Error }\end{array}$ & $\begin{array}{c}10 * 10^{4} \\
\text { Bits }\end{array}$ & $\begin{array}{c}\% \\
\text { Error }\end{array}$ \\
\hline 1 & $5.2290 \mathrm{ps}$ & 4.93 & $5.4800 \mathrm{ps}$ & 0.36 & $5.4900 \mathrm{ps}$ & 0.18 & $5.4730 \mathrm{ps}$ & 0.49 \\
\hline 2 & $5.1890 \mathrm{ps}$ & 5.65 & $5.2540 \mathrm{ps}$ & 4.47 & $5.4180 \mathrm{ps}$ & 1.49 & $5.5400 \mathrm{ps}$ & 0.73 \\
\hline 3 & $5.2760 \mathrm{ps}$ & 4.07 & $5.5970 \mathrm{ps}$ & 1.76 & $5.5200 \mathrm{ps}$ & 0.36 & $5.5300 \mathrm{ps}$ & 0.55 \\
\hline 4 & $5.2296 \mathrm{ps}$ & 4.92 & $5.4822 \mathrm{ps}$ & 0.32 & $5.5071 \mathrm{ps}$ & 0.13 & $5.5105 \mathrm{ps}$ & 0.19 \\
\hline 5 & $5.6880 \mathrm{ps}$ & 3.42 & $5.5899 \mathrm{ps}$ & 1.63 & $5.4457 \mathrm{ps}$ & 0.99 & $5.5116 \mathrm{ps}$ & 0.21 \\
\hline 6 & $5.2441 \mathrm{ps}$ & 4.65 & $5.7223 \mathrm{ps}$ & 4.04 & $5.4562 \mathrm{ps}$ & 0.80 & $5.5014 \mathrm{ps}$ & 0.03 \\
\hline
\end{tabular}

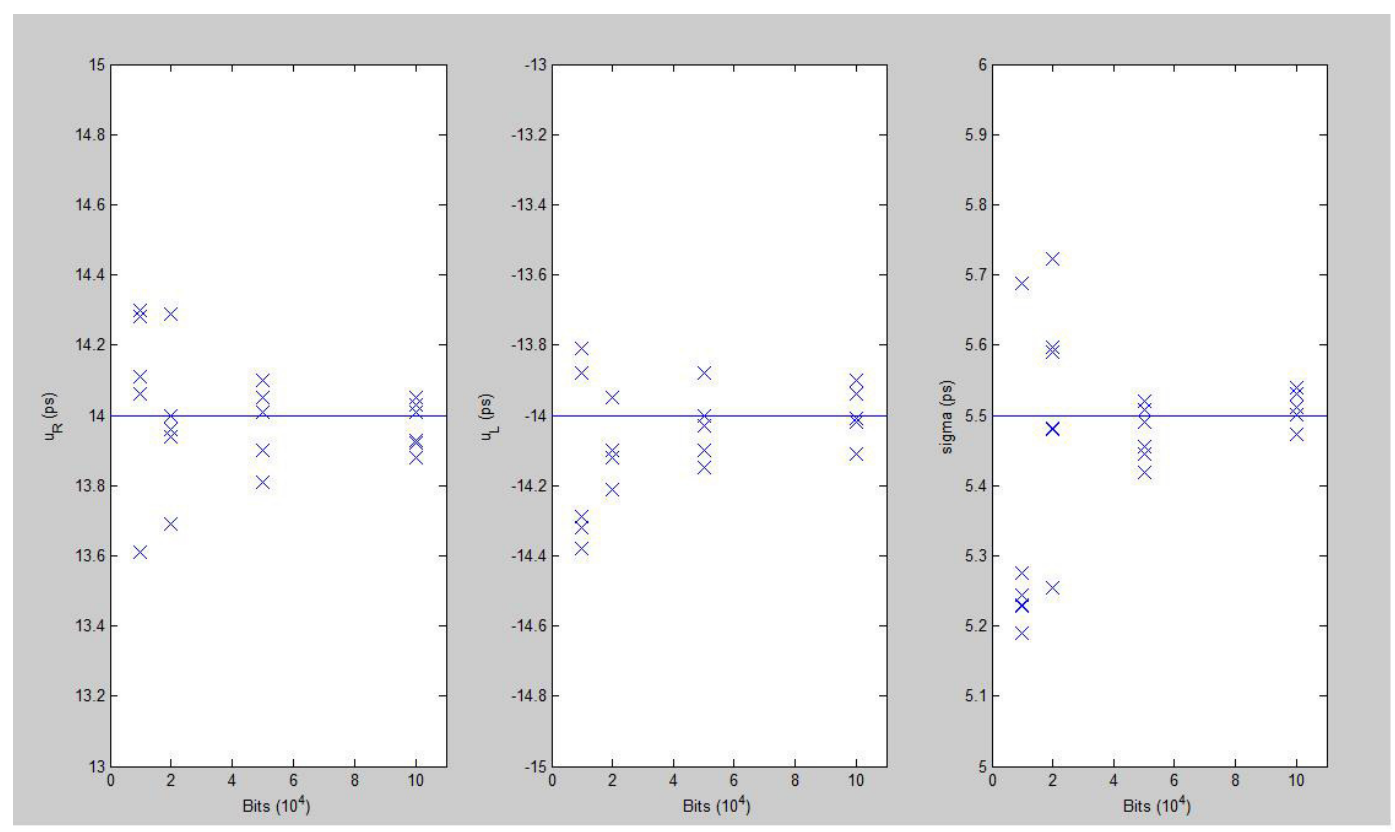

Figure 3.4: Estimation of mean and variance 
Figure 3.4 shows the scattered plot of the data from the Table 3.1, 3.2 and 3.3. The $\mathrm{x}$-axis represents the number of transmitted bits and $\mathrm{y}$-axis represents the spectral information. It clearly shows that variations of estimated TJ spectral information are reduced significantly when larger number samples are captured. The convergence signifies the higher estimation accuracy.

The testing time corresponding to capture $10^{5}$ samples would be $10^{5} /\left(2.5 * 10^{+9}\right)=$ $0.4 * 10^{-4}$ sec when the link runs at $2.5 \mathrm{Gbps}$ (PCIe Generation I). The estimation time for the spectral information using EM algorithm in MATLAB takes 25-30 seconds depending on the PC configuration. However, this time could be much lesser when we will implement EM algorithm in hardware. As a result, we can conclude that the total estimation time is the order of seconds which makes high volume test possible. 


\section{CHAPTER 4}

\section{TIME-TO-DIGITAL CONVERTER}

\subsection{Prior work of Time-to-Digital Converter}

In our proposed BER estimation methodology, TDC is an essential unit. The design and implementation of a TDC for testing high-speed serial links are the most challenging developments in this research work. First, let's review the prior works of TDC for delay measurement. The idea of using the principle of time-todigital conversion for delay measurement is an old one. Some schemes for timeto-digital conversion based on CMOS tapped delay lines that were introduced in [15], [16], [17], [18], [19] and [20]. It included a technique where the delay, to be measured, is converted into a pulse, which in turn is used to charge a capacitor. The final voltage of the capacitor indicates the delay between the two signals. The main drawback is that the leakage of the charge in the capacitor, can cause errors during the test. The other problem is the speed of operation. In multi-gigahertz range, this scheme gives poor performance as to charge up the capacitor properly which results high measurement errors.

An on-chip delay measurement based on timing characterization technique is presented in [22]. The propagation delay of the paths are converted into a pulse using a XOR gate and then sampled with a high-speed clock. A counter is incremented at the edge (positive or negative) of that clock when the output of the XOR gate is 1 . The value of the counter gives the delay. To achieve a good

resolution, say $\frac{1}{100}$, the synchronous clock must run at least 100 times higher than 
the system clock. This is practically impossible when the link is running in Gbps range.

In [23]- [24], a single ended tapped delay line (SEDL) based on CMOS technology is presented. A differential or balanced tapped delay lines or vernier delay line (VDL) along with SEDL are presented in [25]- [26]. A VDL circuit is made of two delay chains. The intermediate tap points are fed to the inputs of a chain of storage elements (which may be the clock and data or S and R input of a flip-flop). The main advantage of the VDL over the SEDL is the resolution of the delay measurement. The resolution the of SEDL is limited by the minimum achievable delay in given technology whereas the resolution of a VDL is determined by the delay difference between the delay elements in the two delay chains. However, the VDL circuit suffers from its size because of the large number of storage elements present in it. Furthermore, the size of the circuit will become larger if higher resolution is required.

\subsection{Proposed Time-to-Digital Converter}

In this research, a novel design of TDC is proposed. The basic concept for the proposed TDC is based on "successive approximation". The digitized output of the converter is set to "1" initially. It remains the same in a particular stage when a successful comparison is made; otherwise, it will be reset to "0". The comparison is carried out bit by bit from the most significant bit (MSB) to least significant bit (LSB).

The proposed design is shown in Figure 4.1. It is basically a single-ended vernier delay line with a buffer chain and associated latching elements. The delays associated with the buffer are in binary format. Thus, if there are $N$ stages, the buffer delays will be $2^{N-1} t_{b u f}, 2^{N-2} t_{b u f}, \ldots, 2^{1} t_{b u f}, 2^{0} t_{b u f}$, where $t_{b u f}$ is the smallest delay available associated a buffer for a given technology. 


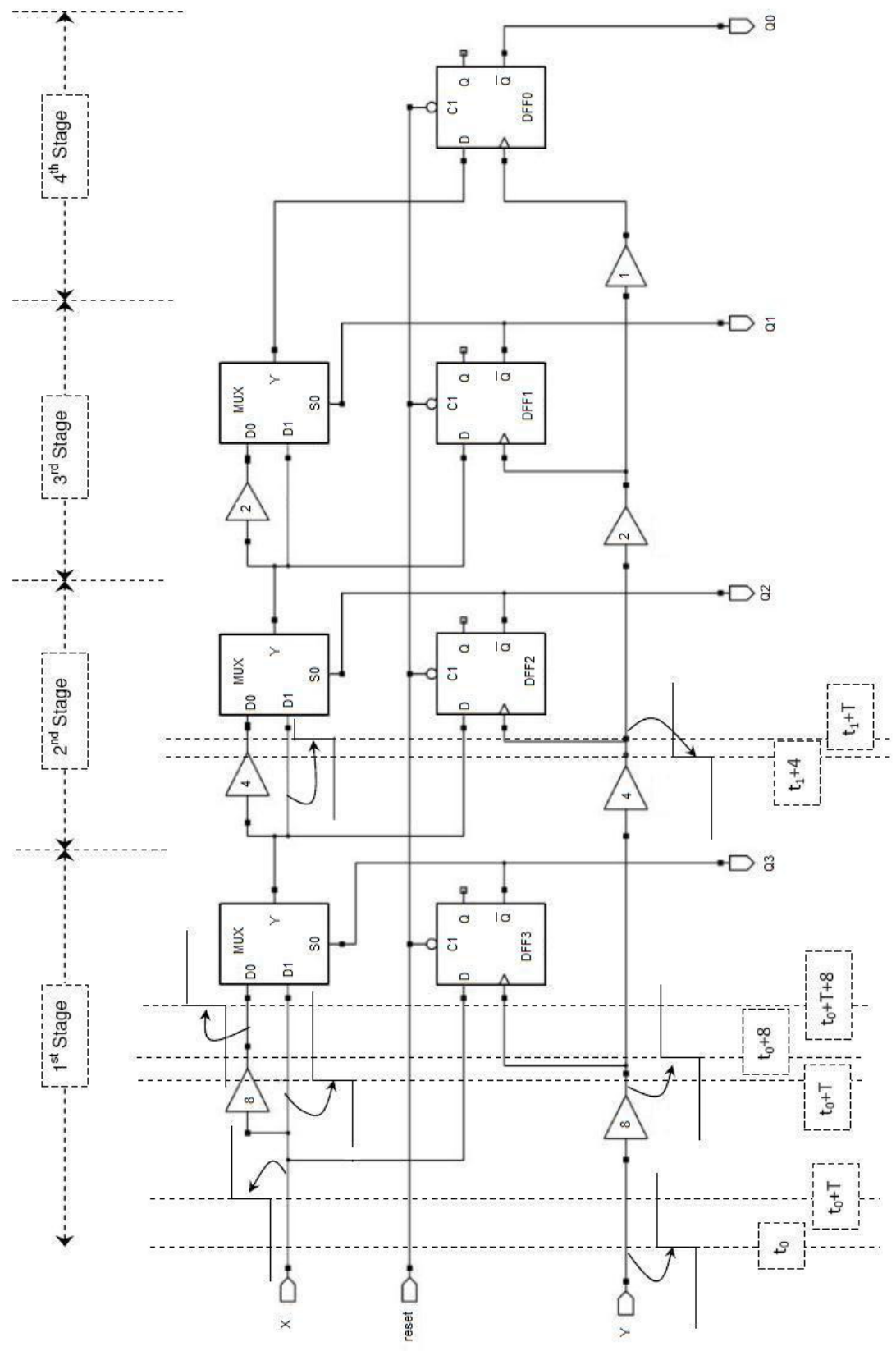

Figure 4.1: Time-to-Digital Converter 
Hence, the resolution of the proposed design is $t_{b u f}$. We can measure a maximum delay of $\left(2^{N-1}+2^{N-2}+\ldots+2^{2}+2^{1}+2^{0}\right) * t_{b u f}$. The measurement of higher delay requires larger number of stages,i.e., larger $\mathrm{N}$.

The upper chain consists of the multiplexers (MUX) and the buffers with the same delay as those in the lower buffer chain. The latching elements are positive edge triggered D flip-flops with asynchronous reset. The data and clock inputs of the flip-flops are coming from the output of the MUX in the previous stage and the output of the lower buffers in the same stage respectively. The output $\bar{Q}$ of the flip-flop is fed to the select input of the MUX in the same stage. $\bar{Q}$ is the final output of this design.

For the simplicity to describe how the design works, we assume all the circuit components except the buffers have no delay. We will discus how the delay of different components affects the operation of this design later in Section 4.4.

Now, let's consider how the delay between the input $\mathrm{X}$ and $\mathrm{Y}$ is to be measured. All the transitions in X and Y must be at rising edges. Before the delay measurement or time-to-digital conversion, all the flip-flops are need to be reset i.e., $Q=0$ and $\bar{Q}=1$. The MUXes of all the stages will select the D1 input. Assuming that the phase difference between $\mathrm{Y}$ and $\mathrm{X}$ (where the transition of $\mathrm{Y}$ comes before the transition of $\mathrm{X}$ ) is $\mathrm{T}$ and our design has four stages with resolution $t_{b u f}=1$ unit. To illustrate how the TDC works, we consider the value of $\mathrm{T}$ is 7 units. At the inputs of TDC, the rising edges of $\mathrm{Y}$ and $\mathrm{X}$ come at $t_{0}$ and $t_{0}+7$ respectively. At stage 1, the output of the lower buffer will have the positive edge transition at $t_{0}+8$, which results the rising edge of the clock comes after the data $(\mathrm{D}=1)$. Thus the output of the flip-flop (DFF3) will be $Q=1$ and $\bar{Q}=0$ and the MUX will select D0 input. As a result, the output of the MUX and the lower buffer has the same phase difference as of $\mathrm{X}$ and $\mathrm{Y}$, i.e., 7 units. In the second stage, the rising edge of the output of the lower buffer comes before the transition of previous stage MUX output that leads the flip-flop (DFF2) remains the same 
state i.e., $Q=0$ and $\bar{Q}=1$. Now, the input phase difference for the stage 3 will be the difference of the input delay of stage 2 to the delay of the lower buffer in stage 2 , i.e., $(7-4)=3$ units. In this stage, this delay is greater than the delay of the lower buffer. As a result, DFF1 will remain the same state i.e., $Q=0$ and $\bar{Q}=1$. Finally, the input phase difference for the stage 4 will be $(3-2)=1$ unit which is equal to the delay of the lower buffer. The flip-flop (DFF0) will remain the same state i.e., $Q=0$ and $\bar{Q}=1$. The final result corresponds to the delay of 7 units between $\mathrm{Y}$ and $\mathrm{X}$ becomes $Q 3 Q 2 Q 1 Q 0=0111$ which is the binary equivalent of 7

Based on the above example, we can observe the following:

- False comparison: In a particular stage, if the delay between the upper and lower chain is less than the delay of the lower buffer, the output from that stage will be " 0 " and it is necessary to add the same buffer delay to the upper chain.

- True comparison: In a particular stage, if the delay between the upper and lower chain is greater than the delay of the lower buffer, the output from that stage will be "1" and the input delay to the next stage will be the difference between the delay input to that stage and the delay of the lower buffer.

\subsection{Simulation Results}

Figure 4.2 shows the Modelsim simulation of the proposed design. Here, the design has four stages and the resolution is set to $1 \mathrm{~ns}$. Initially, all the flip-flops are in reset and the output of the design is 1111. The delay between $\mathrm{Y}$ and $\mathrm{X}$ is 7 ns. The output, corresponding to that delay is 0111 which is the binary equivalent of 7 . 


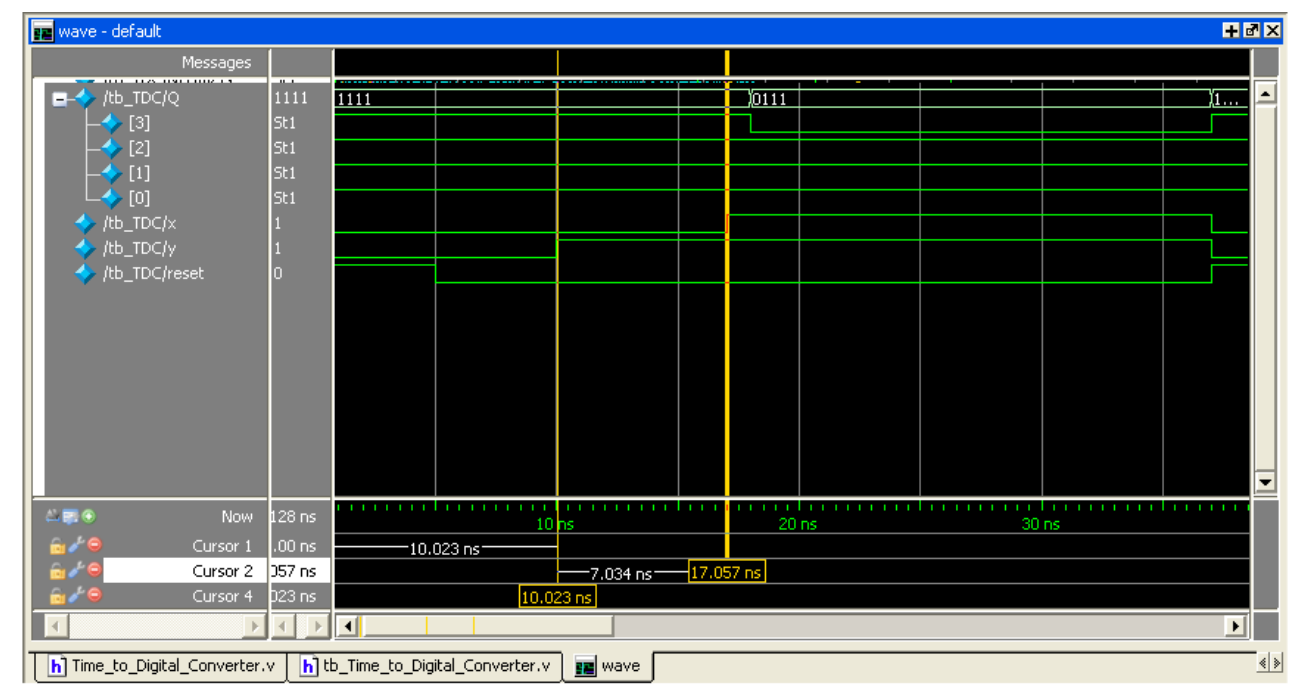

Figure 4.2:

Modelsim simulation waveform of the proposed Time-to-Digital Converter

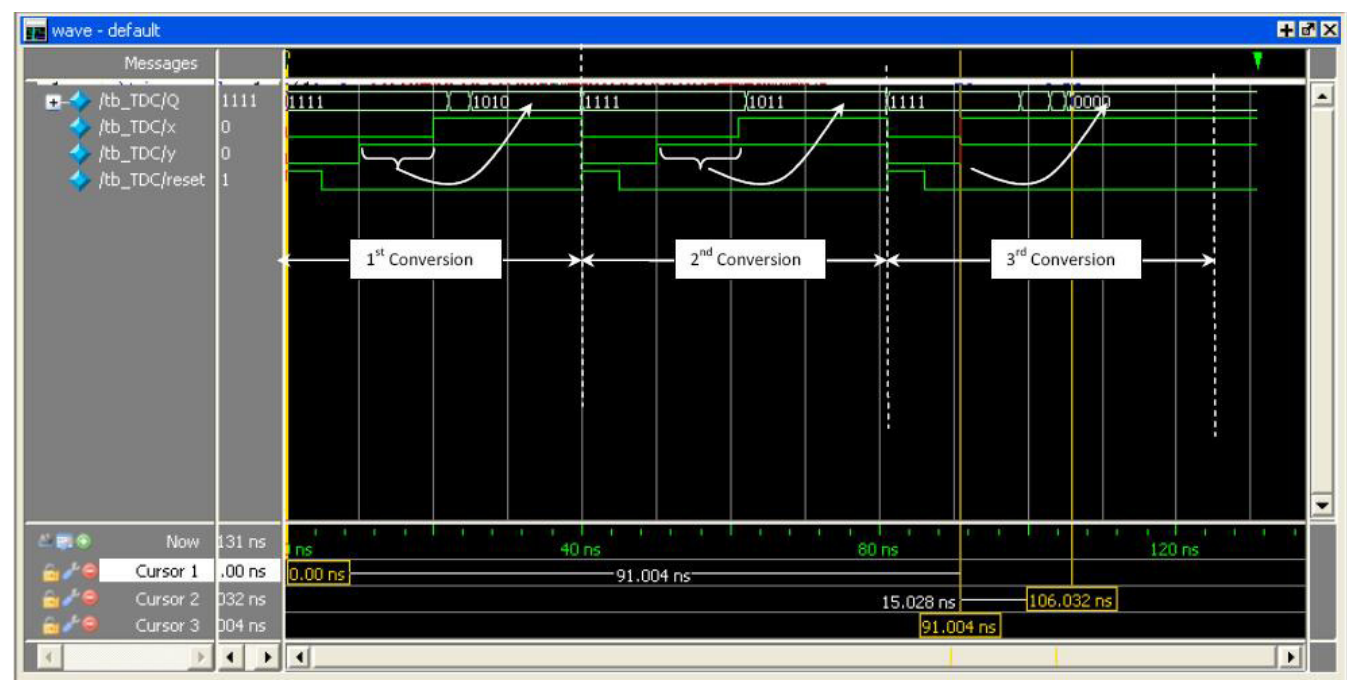

Figure 4.3:

Modelsim simulation waveform of the proposed Time-to-Digital Converter 
Figure 4.3 shows three more measurements of delays with our proposed TDC. In each measurement, the reset pulse is asserted and the output becomes 1111 . In the first conversion, the delay between $\mathrm{Y}$ and $\mathrm{X}$ is $10 \mathrm{~ns}$, the corresponding output is 1010. For the second and third conversions, the delays are 11 ns and 0 ns respectively and the corresponding digital output is 1011 and 0000 respectively. The maximum delay we can measure is $(8+4+2+1)=15$ ns as we have four stages. If we want to measure higher delay, then we have to add more stages to this design.

\subsection{Modifications in the Proposed Design}

Now we will consider the delays in the different components, in addition to the delay of the buffers. Here, the multiplexers (MUX) and the flip-flops (DFFs) contribute different delays to the signal propagation that affects the time-todigital conversion process.

\subsubsection{Buffers}

The minimum buffer delay corresponding to $0.25 \mu$ technology is around 105 ps and the SPICE simulation for the buffers used in this design is shown in the Figure 4.4. The BUF2 has a delay of 210 ps and BUF4 and BUF8 have the delays 420 ps and 840 ps respectively. The minimum buffer delay represents the resolution of the proposed time-to-digital converter. To achieve high resolution, we have to go for newer CMOS technology such as $45 \mathrm{~nm}$ or lower where the minimum buffer delay is 8-10 ps or lower.

\subsubsection{MUX}

The MUX used in our proposed design has two data inputs (D0 and D1) and one selection (S0) input. The output selects D0 or D1 depending on the select 


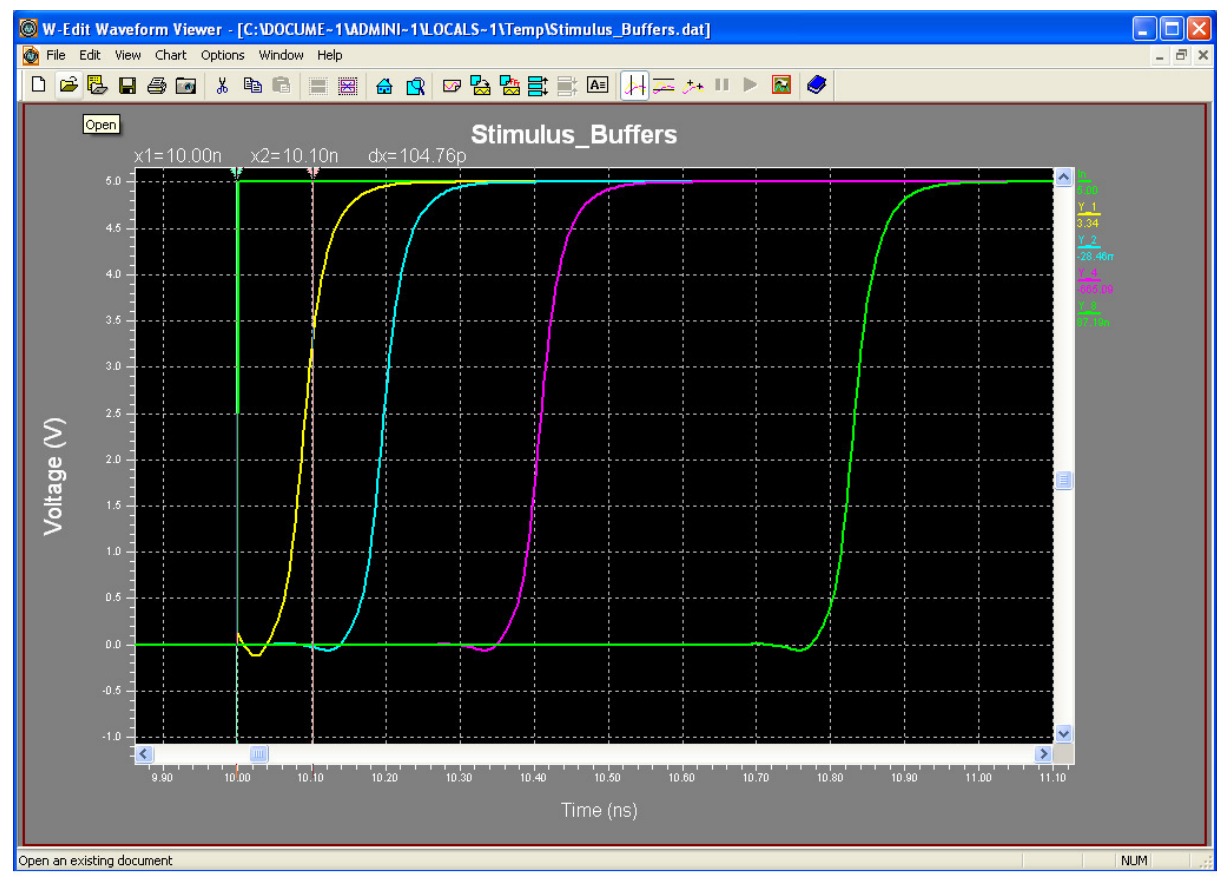

Figure 4.4: The TSPICE simulation for the buffers used in this design 


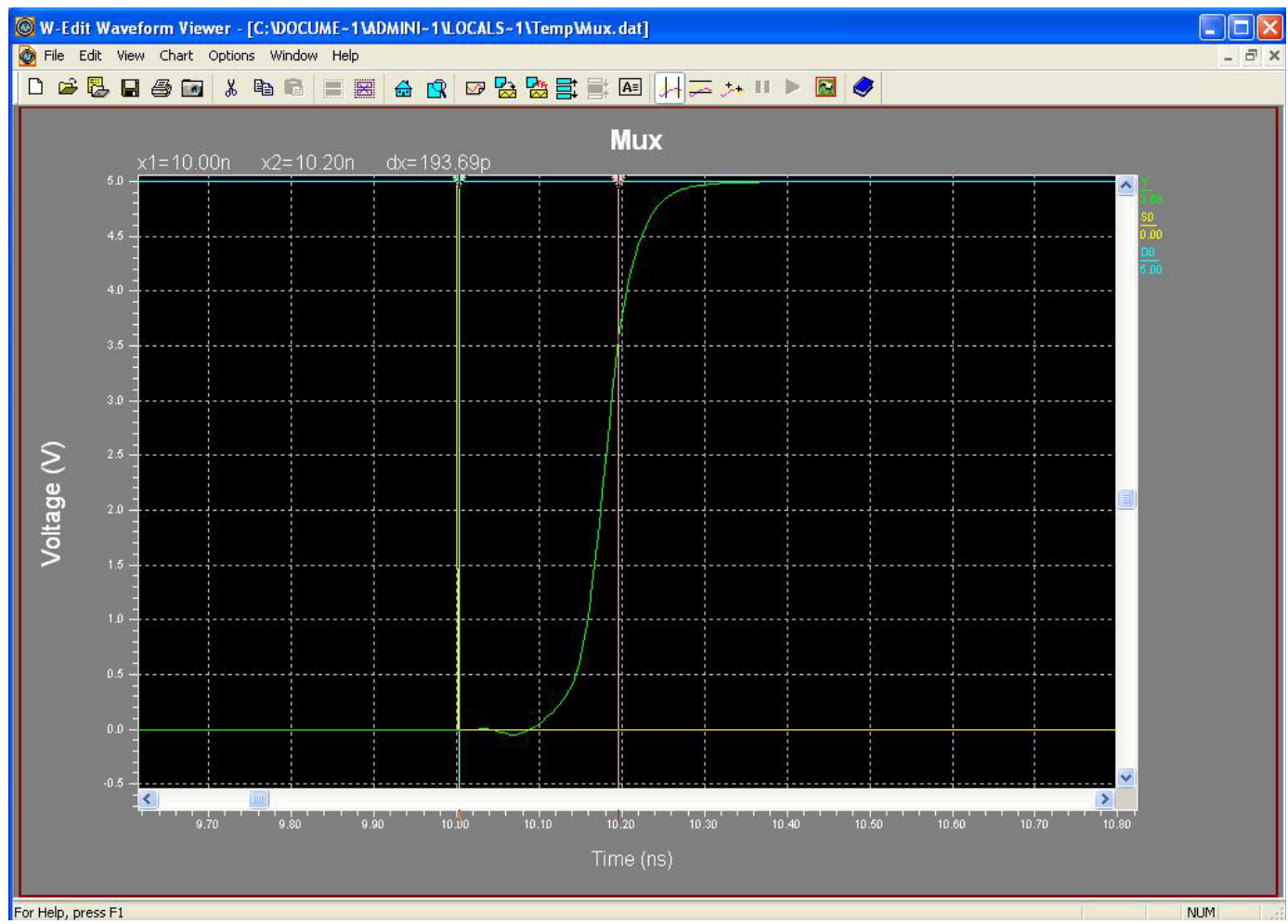

Figure 4.5: TSPICE simulation of MUX

input S0 is 0 or 1 respectively. The output $\mathrm{Y}$ does not change immediately after the change of S0. There will be a definite amount of propagation delay and we are denoting this delay as S-to-Y delay of MUX. Figure 4.5, shows the SPICE simulation of the MUX. To develop the TSPICE model we have selected $0.25 \mu$ technology as the library files corresponding this technology are available in Tanner EDA. The S-to-Y delay of the MUX is around 194 ps.

To compensate the effect of the delay we have to add the same amount of delay (S-to-Y delay of the MUX) by adding a buffer (BUFM) to the lower buffer chain. The Figure 4.6 shows a single stage of of the proposed design after MUX compensation. We have carefully design the buffer to achieve the S-to-Y delay of the MUX. 


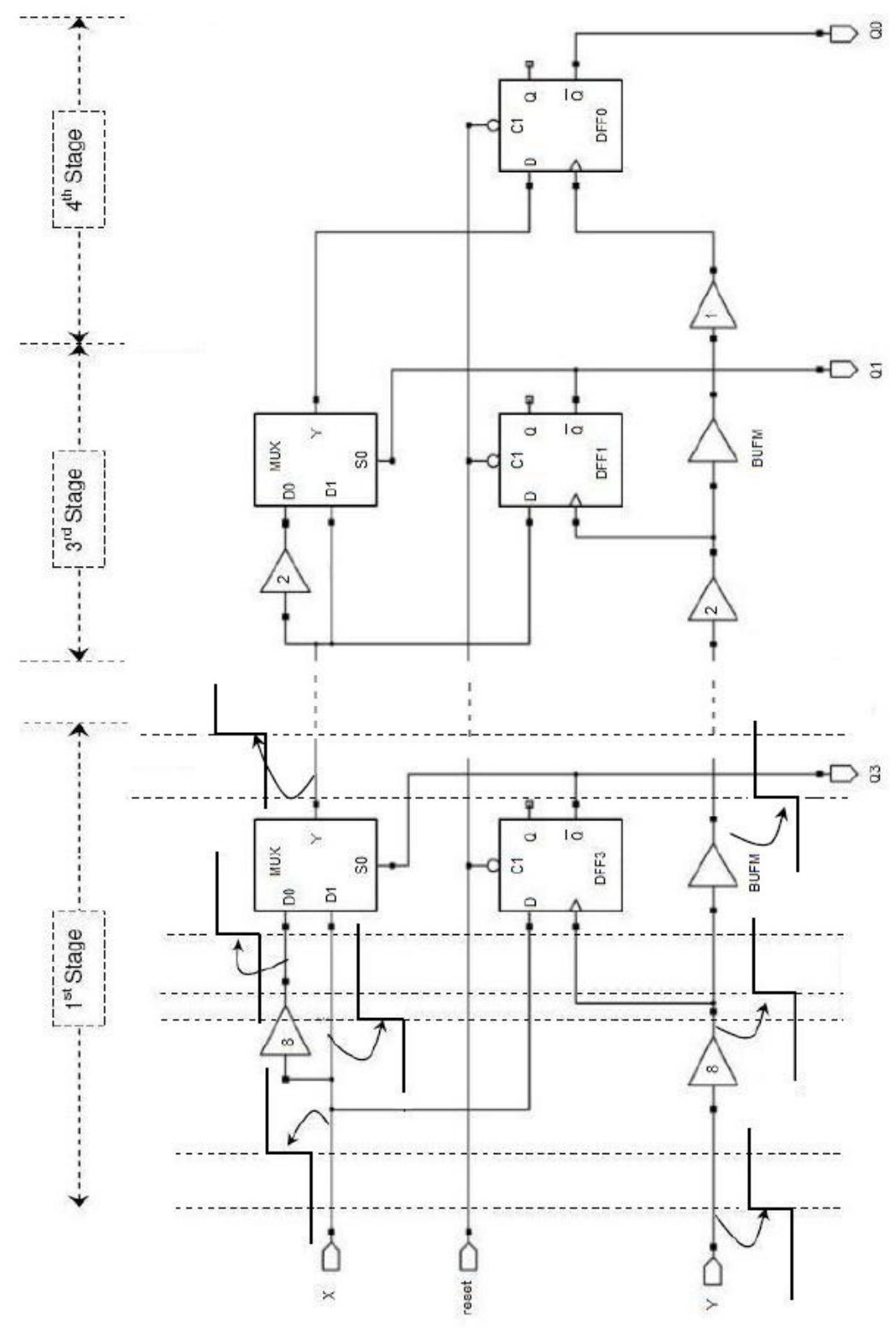

Figure 4.6:

Proposed Time-to-Digital Converter after MUX delay Compensation 


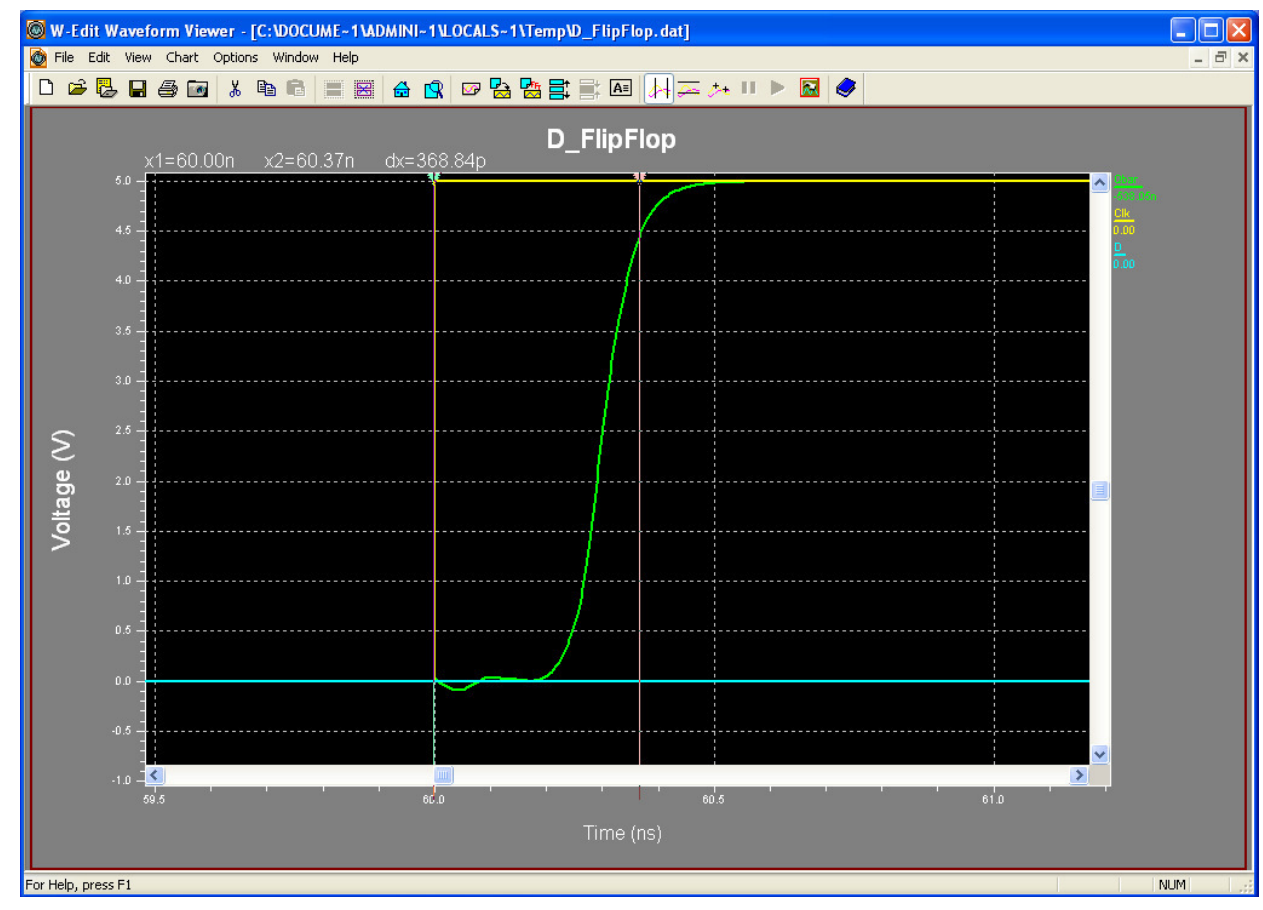

Figure 4.7: TSPICE simulation of DFF

\subsubsection{Flip-flops}

The delay of the flip-flops is generally specified as clock-to- $Q$ delay. Always there will be a time difference between the edge of the clock to the output. However, in our design we have selected $\bar{Q}$ for the selection input of the MUX. Thus, clockto- $\bar{Q}$ delay of the DFFs is considered to nullify the effects of delays in signal propagation. The TSPICE simulation of a DFF is shown in Figure 4.7. The clock-to- $\bar{Q}$ is around 370 ps.

Figure 4.8 shows the modified design of a single stage of the proposed design after the delay compensation of the DFFs. The buffers (BUFD which has delay equal to clock-to- $\bar{Q})$ are added to the upper and lower chain as the clock-to- $\bar{Q}$ delay affects the signal propagation in the upper and lower chains. 


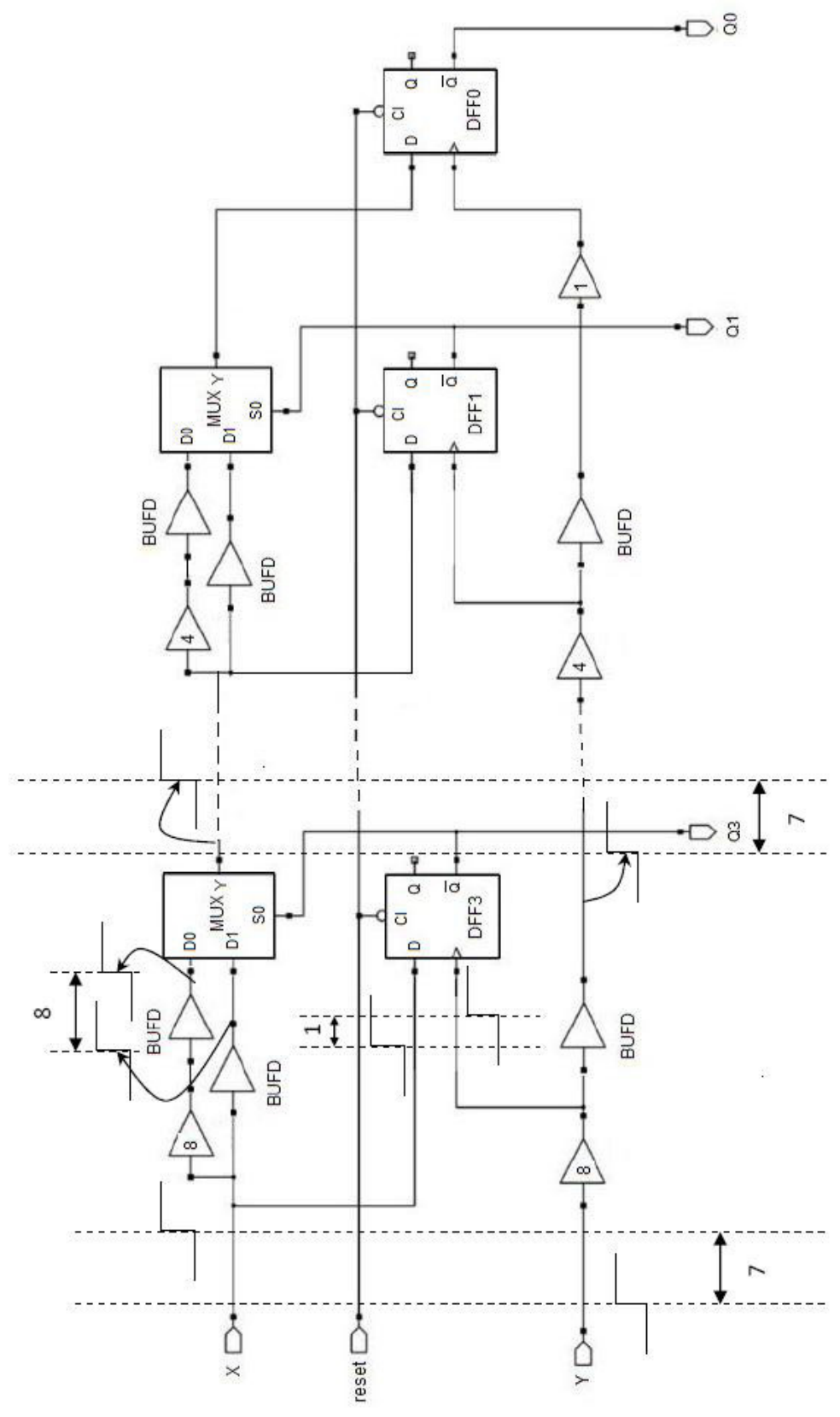

Figure 4.8:

Proposed Time-to-Digital Converter after clock-to- $\bar{Q}$ delay Compensation 


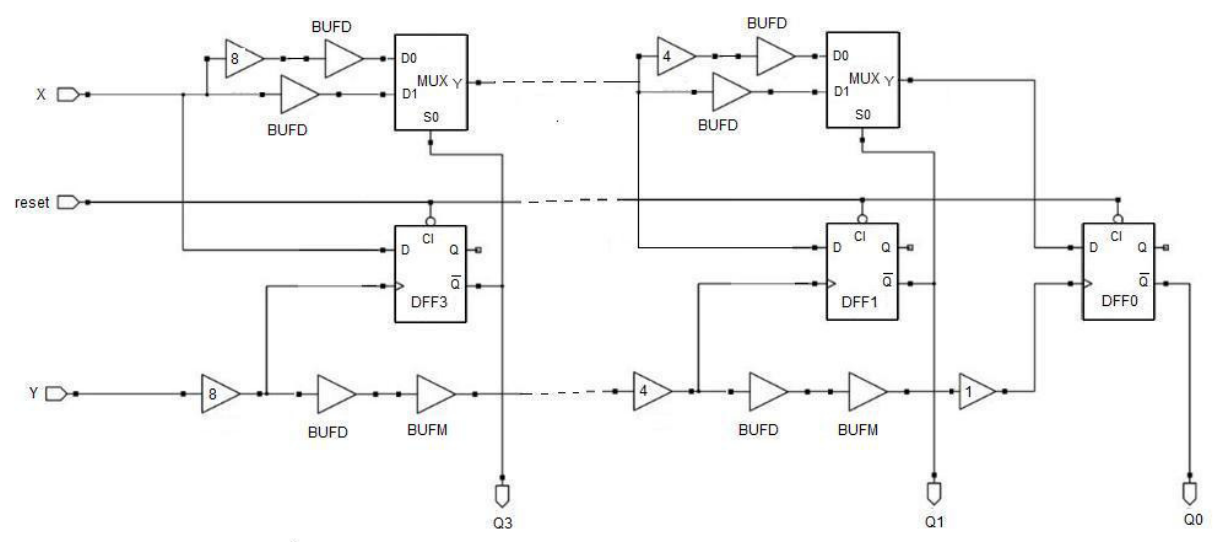

Figure 4.9: The complete proposed Time-to-Digital Converter

\subsubsection{Final Design and Simulation}

Figure 4.9 shows the final design after the compensation of the S-to-Y delay of the MUX and clock-to- $\bar{Q}$ delay of the DFFs.

Figure 4.10 shows the TSPICE simulation of the proposed Time-to-Digital Converter. We have implemented this design in TSPICE with .25 $\mu$ technology. The resolution of this simulation is 105 ps because of the lowest buffer delay available $.25 \mu$ technology. In this simulation, we set the delay between $\mathrm{Y}$ and $\mathrm{X}$ is $1200 \mathrm{ps}$. The first comparison is true as $1200 \mathrm{ps}$ is greater than $840 \mathrm{ps}$, the second comparison is false as $(1200-840)=360$ ps is less than 420 ps, the third comparison is true as 360 ps is greater than 210 ps and the final comparison is true as $140 \mathrm{ps}$ is greater than $105 \mathrm{ps}$. The final outputs Q3=1 $(5 \mathrm{~V}), \mathrm{Q} 2=0(0$ $\mathrm{V}), \mathrm{Q} 1=1(5 \mathrm{~V}), \mathrm{Q} 0=1(5 \mathrm{~V})$. The result clearly verifies the proper operation of our proposed TDC. 


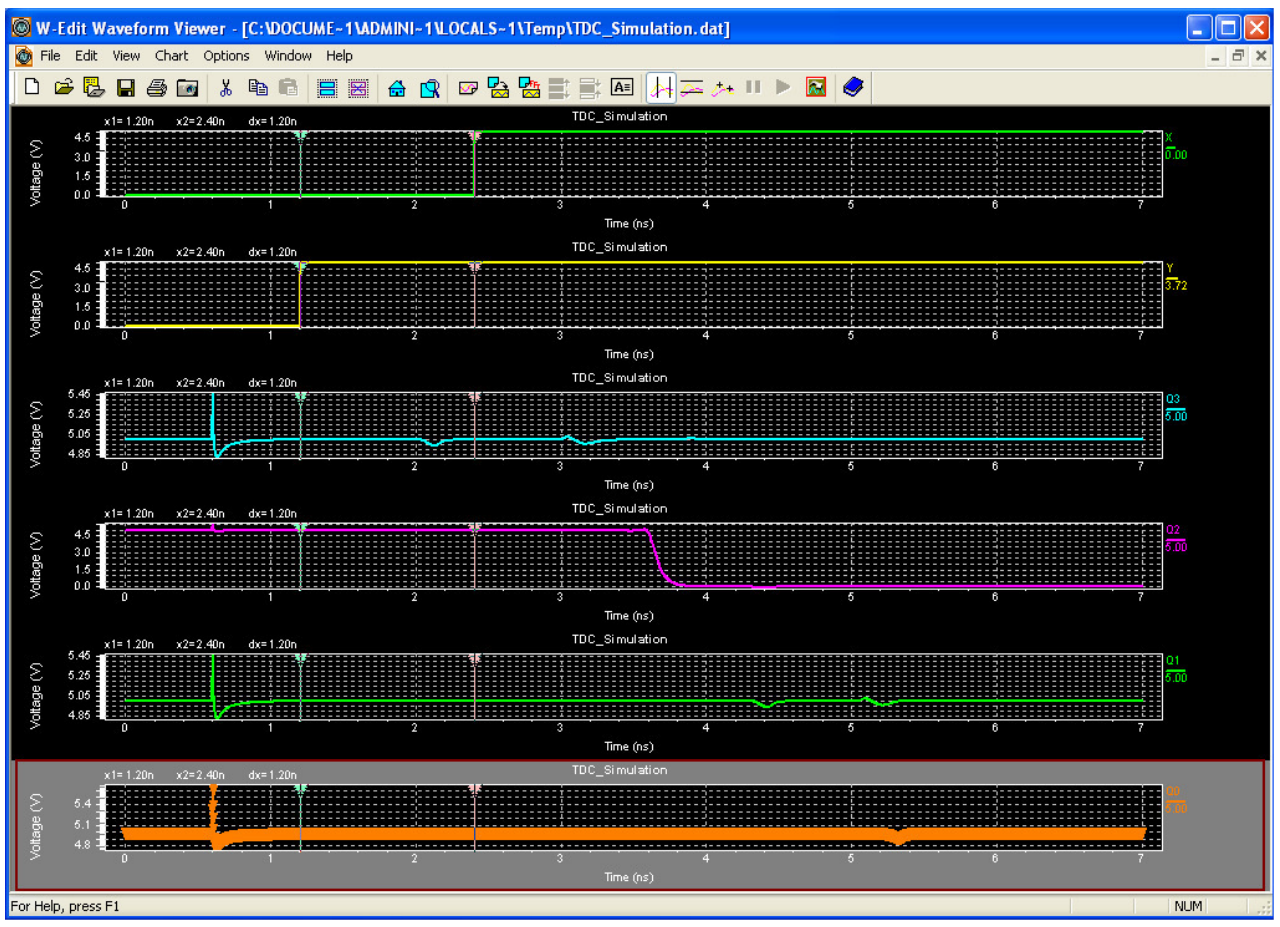

Figure 4.10:

The TSPICE simulation for the proposed Time-to-Digital Converter 


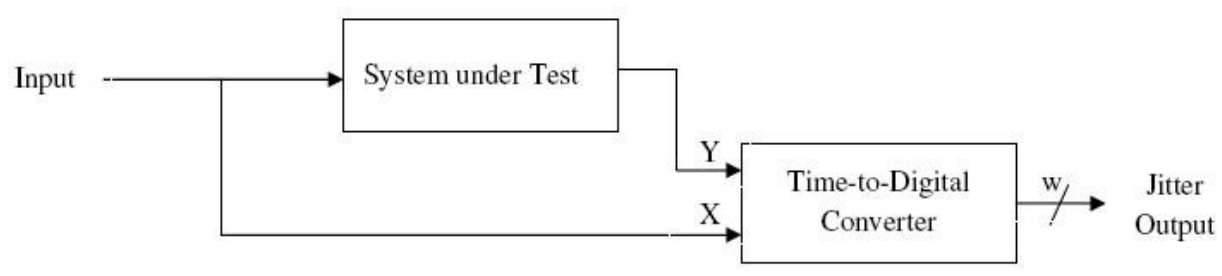

Figure 4.11: Set-up for the jitter measurement of a system

\subsection{Application: Jitter Measurement}

This proposed time-to-digital converter can measure the jitter generated in a system. Figure 4.11 shows the jitter measurement set-up. The system under test (SUT) can be the receiver of a SerDes. It is important to know how much jitter is generated in the receiver for its characterization. This is also true for the characterization of the transmitter of a SerDes.

The jitter free serial data is given to he input of the set-up. Due to the generation of the jitter in the SUT, the output of it will be the data embedded with jitter. The jitter free and the jittery data will be the two inputs of our proposed TDC. The output of the TDC will directly give the jitter generated in the system. The resolution of the measurement process entirely depends on the resolution of the TDC. 


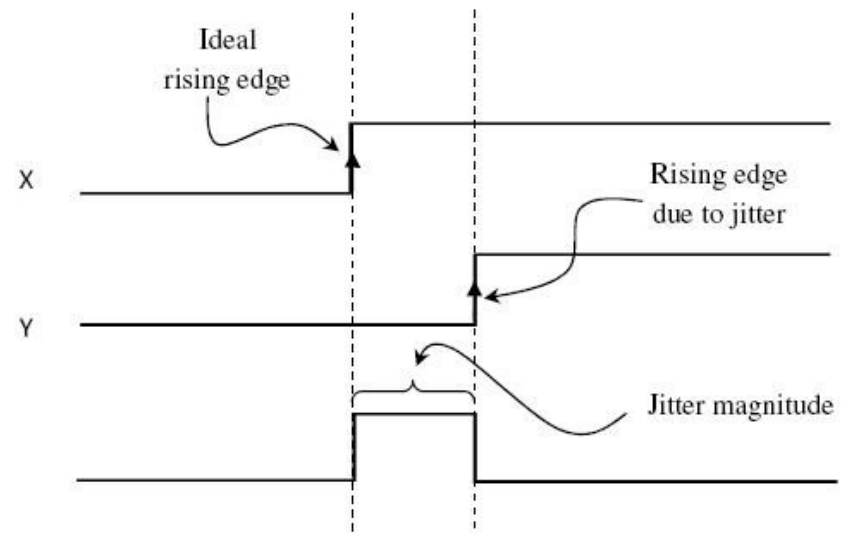

Figure 4.12: Jitter measurement using our proposed Time-to-Digital Converter

Figure 4.12 describes the jitter measurement process. The delay between the rising edges of jittery and jitter free data is measured with our proposed TDC. It represents the jitter magnitude. 


\section{CHAPTER 5}

\section{CONCLUSION}

\subsection{Contributions}

In this research, we have proposed a novel BER estimation method. To implement that proposed method, we have designed a new time-to-digital converter. Our research has the following contributions,

- The proposed BER estimation method makes it possible to measure BER accurately without the prior knowledge of jitter present in the serial data stream. This is the significant improvement over the dual-Dirac model.

- The novel time-to-digital converter directly converts the delay between the two signals in the digital format. The significant improvement over its counterpart is the improved resolution. We can measure delay in the range of picoseconds. Also, the resolution of the design could be varied with selecting different process technology.

- The jitter, present on the serial data, could be measured very accurately with the proposed time-to-digital converter as it measures the delay between the signals with fine resolution. 


\subsection{Conclusion}

An efficient test methodology for the high-speed serial links is developed in this research. In Chapter 2, the background study of SerDes and various types of jitters present in the serial data have been done. We have studied two popular BER estimation methods. The drawbacks for the estimation of BER have been discussed.

A novel BER estimation method has been presented in Chapter 3. This method incorporates the comparison of jitter spectral information of the golden SerDes with the test one. The spectral information of the total jitter, solely responsible for bit errors, is successfully estimated by using MATLAB simulation. We have developed a complete design for the BER estimation.

To implement the proposed BER estimation methodology, we have proposed a novel time-to-digital converter in Chapter 4. This design successfully converts the delay between two signals into its digital counterpart. The design and its SPICE simulation have been presented. 


\subsection{Future Work}

We have proposed an efficient BER estimation method for the high volume testing of SerDes. This method efficiently estimates the TJ spectral information of the serial data. We have validated the estimation of the spectral information by MATLAB simulation in Chapter 3. However, we couldn't estimate the TJ spectral information of the recovered clock due to unavailability of the spectral information for the clock in the literature. Our future research will be directed to obtain that information by measuring it from a SerDes and estimate the BER.

To implement the proposed design, we have proposed a novel time-to-digital converter. The SPICE simulation in Chapter 4 confirms the result. The resolution of this solely depends on the process technology. We can achieve a resolution of 8-10 ps for $45 \mathrm{~nm}$ technology. However, further increase in resolution requires lower process technology. The possible solution might be the modification of the proposed time-to-digital converter which uses a VDL to a converter with a differential VDL. This would be fruitful for further research area.

The another task is to design the BER estimator for the implementation of the proposed BER method. EM Algorithm for the estimation of mean and variance of the total jitter distribution and the computation of the Equation 3.3 would be implemented in the same chip along with the Time-to-Digital converter. The proposed BER estimation method could be implemented in Vertex6 FPGA as it is manufactured with 40 and $45 \mathrm{~nm}$ process. The resolution of the time-to-digital converter would be in the range of picoseconds and could measure jitter with a fine resolution. This could be another research area to be focused. 


\section{REFERENCES}

[1] A. Athavale and C. Christensen, High-Speed Serial I/O Made Simple A Designers' Guide, with FPGA Applications, Connectivity Solution, Edition 1.0, Xilinx.

[2] Agilent Technologies, Using Clock Jitter Analysis to Reduce BER in Serial Data Applications, Available at www.agilent.com

[3] Y. Cai, T. P. Warwick, S. G. Rane and E. Masserrat, Digital Serial Communication Device Testing and its Implications on Automatic Test Equipment Architecture, International Test Conference, 2000, pp. 600.

[4] W. P. Robins, Phase Noise in Signal Sources (Theory and Application), IEE Telecommunications Series, ISBN-10: 086341026X.

[5] William R. Eisenstadt, Bob Stengel, Bruce M. Thompson, Microwave Differential Circuit Design Using Mixed Mode S-Parameters, ISBN-10: 1580539335 .

[6] D. B. Sullivan, D. W. Allan, D. A. Howe, F. L. Walls, Characterization of Clocks and Oscillators, NIST Technical Note 1337, 1990.

[7] J. D. H. Alexander, Clock recovery from random binary data, Electronics Letters, vol. 11, Oct. 1975, pp. 541-542.

[8] J. Lee et al., Analysis and Modeling of Bang-Bang Clock and Data Recovery Circuits, IEEE Journal of Solid State Circuits, Vol. 39, No. 9, Sep. 2004, pp. 1571-1580. 
[9] Y. Choi et al., Jitter Transfer Analysis of Tracked Oversampling Techniques for Multigigabit Clock and Data Recovery, IEEE Transaction on Circuits and Systems II, Vol. 50, No. 11, Nov. 2003, pp. 775-783.

[10] Behzard Razavi, Design of Analog CMOS Integrated Circuits, Tata McGraw-Hill Edition, ISBN-0-07-052903-5.

[11] Agilent Technologies, Jitter Analysis: The dual-Dirac Model, RJ/DJ, and Q-scale, Available at www.agilent.com

[12] D. Hong et al, Bit Error Rate Estimation for High Speed Serial Links, IEEE Transaction on Circuits and Systems I, Vol. 53, No. 12, Dec. 2006, pp. 2616-2627.

[13] Agilent Technologies, Total Jitter Measurement at Low Probability Levels, Using Optimized BERT Scan Method, Available at www.agilent.com

[14] Y. Cai et al, Jitter Testing for Multi-Gigabit Backplane SerDes Techniques to Decompose and Combine Various Types of Jitter, Proceedings of International Test Conference, Nov. 2002, pp. 700-709.

[15] M. Li, and J. Wilstrup, Paradigm Shift for Jitter and Noise in Design and Test $>1 \mathrm{~Gb} / \mathrm{s}$ Communication Systems, IEEE Proceedings of International Conference on Computer Design, Oct. 2003, pp. 467-472

[16] J. Christiansen, An Integrated CMOS 0.15ns Digital Timing Generator for TDCs and Clock Distribution Systems, IEEE Transactions on Nuclear Science, Vol. 42, Issue: 4, August 1995, pp. August 1995.

[17] M. S. Gorbics, J. Kelly, K. M. Roberts, and R. L. Sumner, A HighResolution Multi-Hit Time-to-Digital Converter Integrated Circuit, IEEE Transactions on Nuclear Science, Vol. 44, Issue: 3, June 1997, pp. 379-384. 
[18] C. T. Gray, W. Liu, W. A. V. Noije, T. A. Hughes, and R. K. C. III, A Sampling Technique and its CMOS Implementation with $1 \mathrm{~Gb} / \mathrm{s}$ Bandwidth and 25 ps Resolution, IEEE Journal of Solid State Circuits, Vol. 29, Issue: 3, Mar 1994, pp. 340-349.

[19] C. Ljuslin, J. Christiansen, A. Marchioro, and O. Klingsheim, An Integrated 16-channel CMOS Time to Digital Converter, IEEE Transactions on Nuclear Science, Vol. 41, Issue: 4, August 1994, pp. 1104-1108.

[20] T. Rahkonen and J. T. Kostamovaara, The Use of CMOS Delay lines for Digitization of Short Time Intervals, IEEE Journal of Solid State Circuits, Vol. 28, Issue: 8, August 1993, pp. 887-894.

[21] A. Stevens, R. P. Vanberg, J. V. D. Spiegel, and H. H. Williams, A Timeto-Voltage Converter and Analog Memory for Colliding Beam Detectors, IEEE Journal of Solid State Circuits, Vol. 24, Issue: 6, December 1989, pp. 1748-1752.

[22] C. Su, Y. T. Chen, M. J. Huang, G. N. Chen, and C. L. Lee., All digital built-in delay and crosstalk measurement for on-chip buses, Design, Automation, and Test in Europe, IEEE, March 2000, pp. 527-533.

[23] M. Bazes, A novel precision MOS synchronous delay line, IEEE Journal of Solid State Circuits, Vol. 20, Issue: 6, Dec 1985, pp. 1265-1271.

[24] M. Bazes and R. Ashuri, A Novel CMOS Digital Clock and Data Decoder, IEEE Journal of Solid State Circuits, Vol. 27, Issue: 12, December 1992, pp. 19341940.

[25] J. F. Genat, High resolution time-to-digital converters, Nuclear Instruments and Methods in Physics Research, Section A, Vol. 315, Issue 1-3, pp. 411414. 
[26] J. F. Genat and F. Rossel, Ultra High-Speed Time-to-Digital Converter, United States Patent no. 4719608, January 1988.

[27] S. Borman, The Expectation Minimization Algorithm A short tutorial, July 2004.

[28] C. Tomasi, Estimating Gaussian Mixture Densities with EM - A Tutorial [29] www.mathworks.com

[30] www.agilent.com 


\title{
APPENDIX A
}

\section{MATLAB CODES FOR THE TJ SPECTRAL}

\section{INFORMATION ESTIMATION}

\author{
A.1 Total Jitter \\ function TJ=bimodel(u1, sigma1, u2, sigma2) \\ if (rand $\geq 0.5)$ \\ $\mathrm{TJ}=\mathrm{u} 1+\operatorname{sqrt}($ sigma 1$) * \operatorname{randn} ;$ \\ else \\ $\mathrm{TJ}=\mathrm{u} 2+\operatorname{sqrt}(\operatorname{sigma} 2) * \operatorname{randn} ;$ \\ end
}

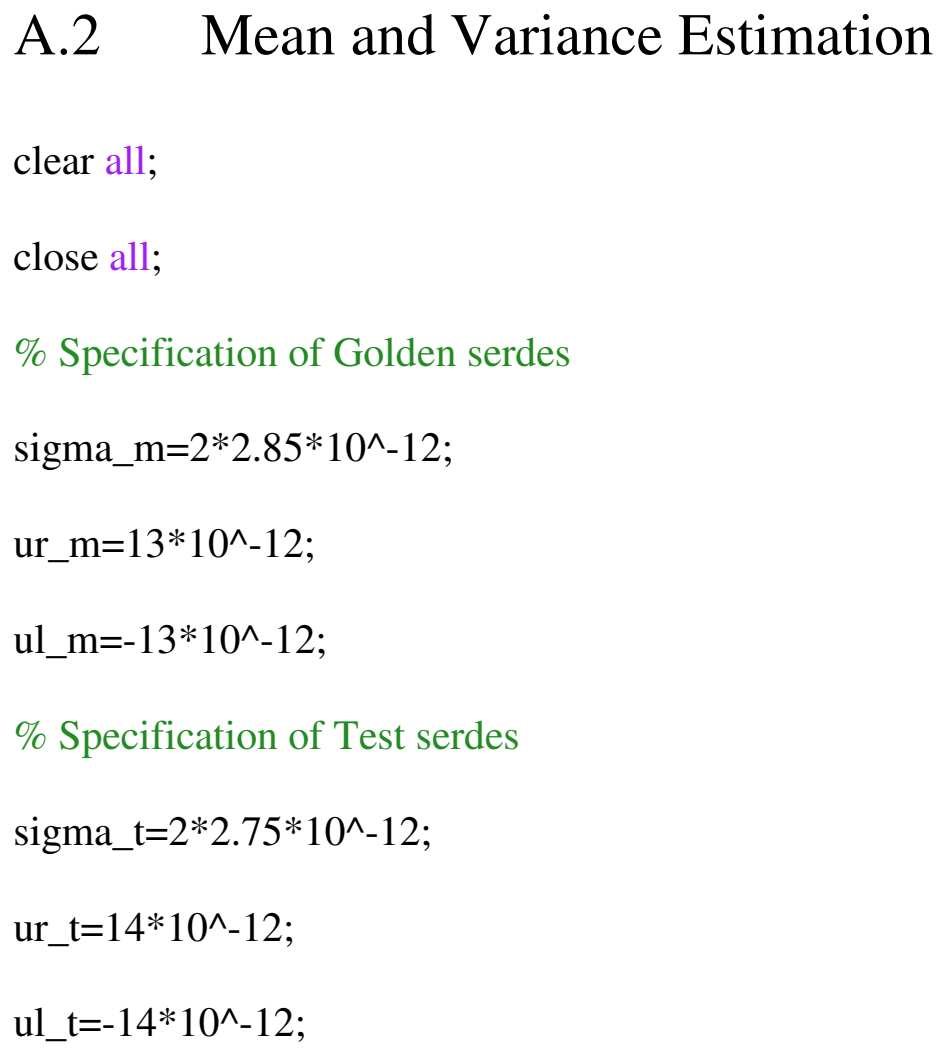

A.2 Mean and Variance Estimation

clear all;

close all;

$\%$ Specification of Golden serdes

sigma $\_\mathrm{m}=2 * 2.85 * 10^{\wedge}-12$;

ur_m $=13 * 10^{\wedge}-12$;

ul_m=-13*10^-12;

$\%$ Specification of Test serdes

sigma_t $=2 * 2.75 * 10^{\wedge}-12$;

ur_t $=14 * 10^{\wedge}-12$;

ul_t=-14*10^-12; 
$\%$ Generation of Total Jitter Distribution

len $=5 * 10000$

for $\mathrm{i}=1$ :len

$\mathrm{y}(\mathrm{i})=$ bimodel $\left(\mathrm{ul} \_\mathrm{m}\right.$, sigma_m,ur_m, sigma_m); $\quad$ \%Distribution of the Model

Serdes (Y)

$\mathrm{x}(\mathrm{i})=$ bimodel $\left(\mathrm{ul} \_\mathrm{t}, \operatorname{sigma} \mathrm{t}, \mathrm{ur} \_\mathrm{t}, \operatorname{sigma} \_\mathrm{t}\right) ; \quad \%$ Distribution of the Test Serdes $(\mathrm{X})$

end

$\% \%$ Plotting the Distribution

$\mathrm{y} 1=\operatorname{hist}(\mathrm{y}, 1000)$;

$\mathrm{x} 1=\operatorname{hist}(\mathrm{x}, 1000)$;

figure(1)

subplot $(3,1,1)$

$\operatorname{hist}(\mathrm{y}, 1000)$;

title('Total Jitter Distribution of Golden Serdes');

xlabel('Time ( Sec )');

$\operatorname{axis}\left(\left[-6^{*} 10^{\wedge}-116^{*} 10^{\wedge}-110400\right]\right)$;

grid on

$\operatorname{subplot}(3,1,2)$

hist(x,1000);

title('Total Jitter Distribution of Test Serdes');

xlabel('Time ( Sec )');

$\operatorname{axis}\left(\left[-6^{*} 10^{\wedge}-116^{*} 10^{\wedge}-110400\right]\right)$; 
grid on

$\mathrm{Z}=\mathrm{x}-\mathrm{y}$

$\operatorname{subplot}(3,1,3)$

$\mathrm{z}=\operatorname{hist}(\mathrm{Z}, 1000)$;

$\operatorname{hist}(Z, 1000)$;

title('Resultant Jitter Distribution');

xlabel('Time ( Sec )');

$\operatorname{axis}\left(\left[-6^{*} 10^{\wedge}-116^{*} 10^{\wedge}-110400\right]\right)$;

grid on

$\% \%$ Estimation of the Mean and Variance of the Test SerDes

$[\mathrm{u}$, sig,t,iter $]=$ mix_gaussian_fitting $\left(\mathrm{Z}^{\prime}, 3\right)$;

sigma_x=sqrt(sig.^2-sigma_m^2*ones(1,length(sig)));

sigma_x=sigma_x(1)

$\mathrm{u} \_\mathrm{x}=\left[-\mathrm{ul} \_\mathrm{m}+\mathrm{u}(1) \quad-\mathrm{ur} \_\mathrm{m}+\mathrm{u}(3)\right]$ 


\section{A.3 EM Algorithm [29]}

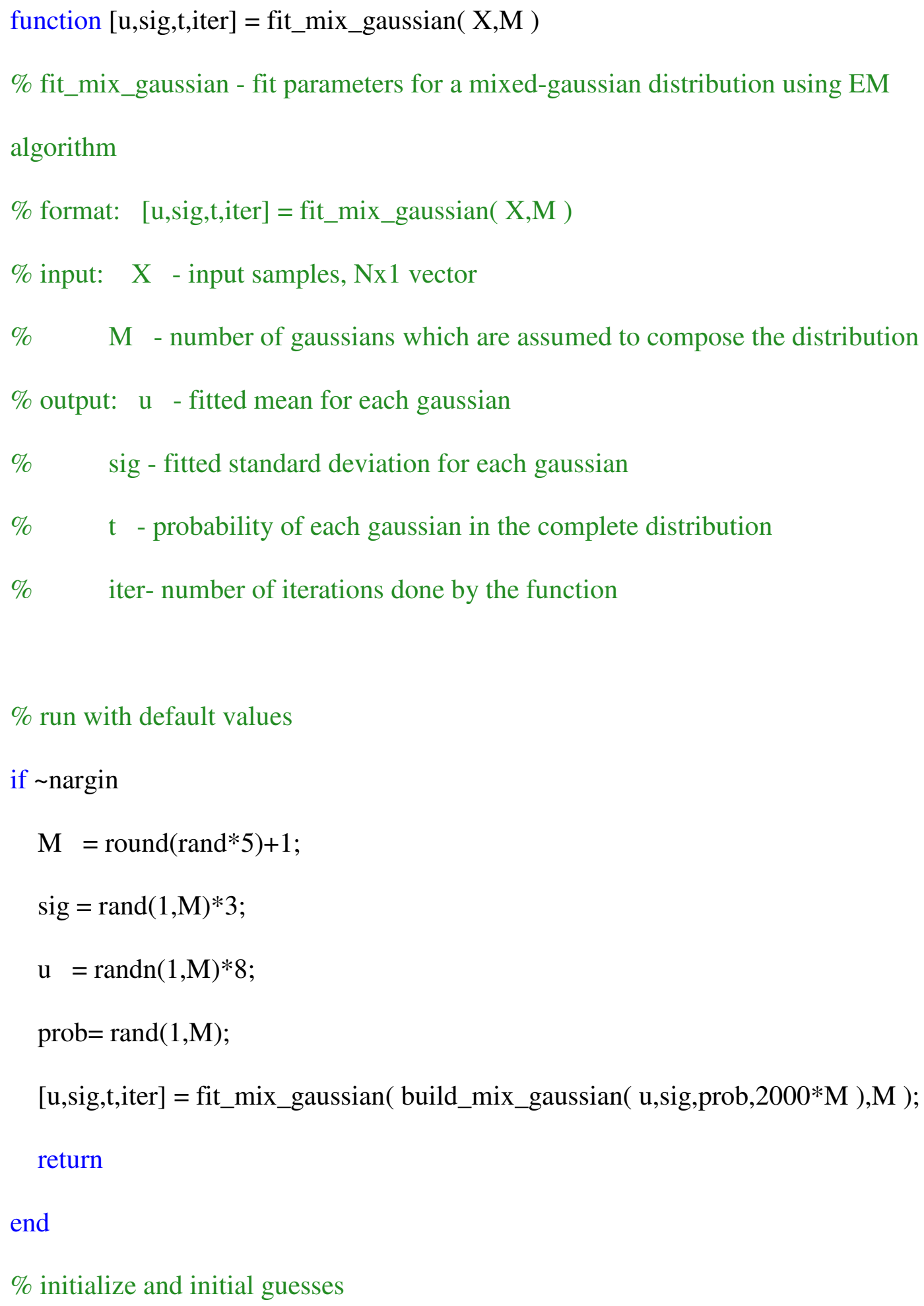




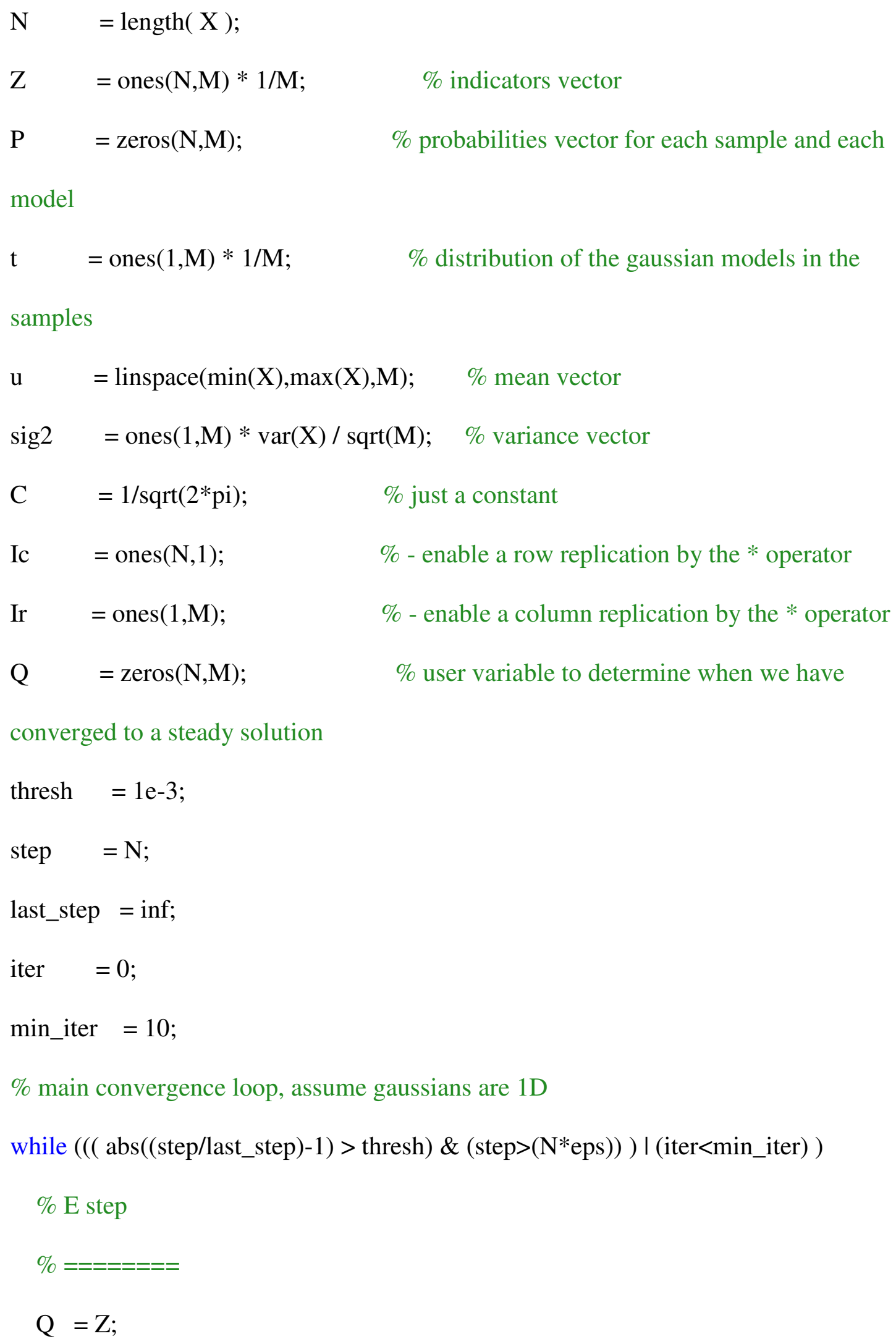




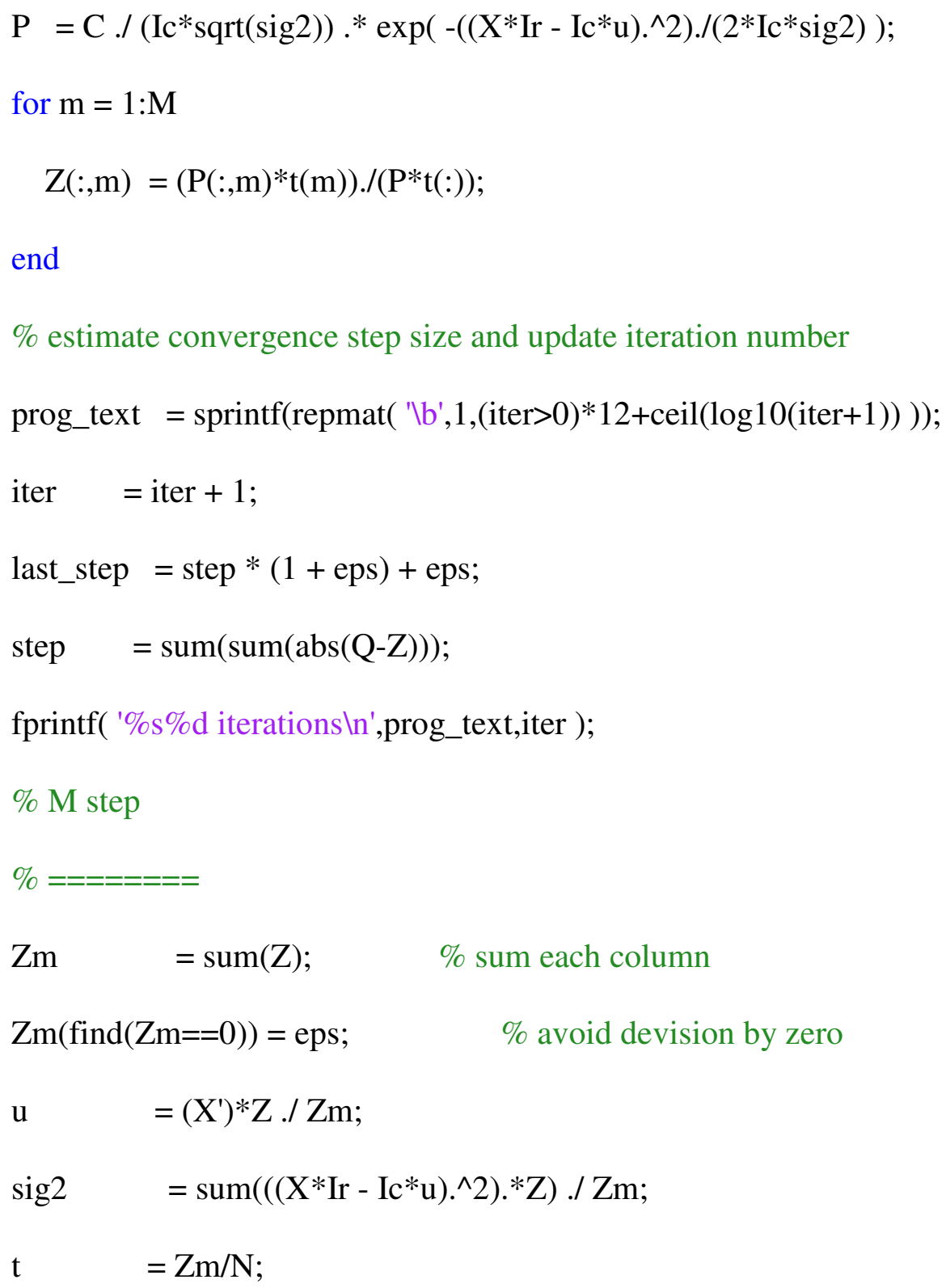




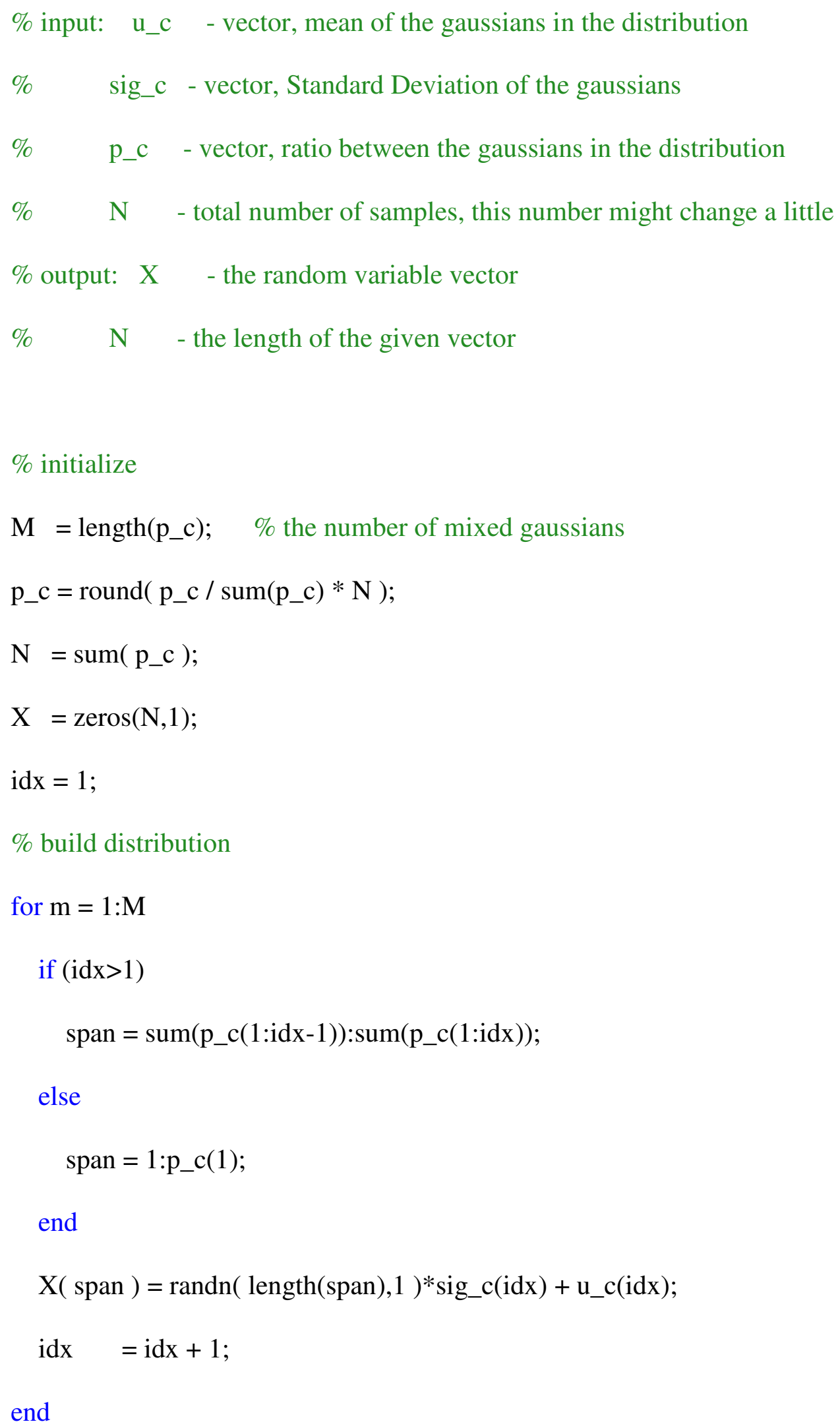




\section{APPENDIX B}

\section{TSPICE CODE FOR THE PROPOSED TIME-}

\section{TO-DIGITAL CONVERTER}

* Design: $\quad$ Time to Digital Converter

* Cell: $\quad$ TDC_Simulation

* View: TDC_Simulation

* Root path: $\quad$ C:ITannerlThesis\Time to Digital ConverterlTime to Digital

Converter

* Exclude global pins: no

* Control property name: SPICE

********** Simulation Settings - General section $* * * * * * * * *$

.option search="C:\Documents and Settings\Administrator $\backslash M y$ Documents $\backslash$ Tanner

EDAlTanner Tools v13.0 LLibraries\Models TT"

.lib "C: IDocuments and Settings\Administrator\My Documents\Tanner EDA\Tanner

Tools v13.0\Libraries\Models\Generic_025.lib" TT

$* * * * * * * * * * * * * * *$ Subcircuits $* * * * * * * * * * * * * * * * *$

.subckt Buf1 In Out Gnd Vdd

*-------- Devices: SPICE.ORDER < 0 --------

* Design: LogicGates / Cell: Buf1 / View: Main / Page: 
* Designed by: Tanner EDA Library Development Team

* Organization: Tanner EDA - Tanner Research, Inc.

* Info: 1x Buffer

*-------- Devices: SPICE.ORDER > 0 --------

MN1 1 In Gnd Gnd NMOS W=2.5u L=250n AS=2.25p PS=6.8u AD=2.25p PD=6.8u MN2 Out 1 Gnd Gnd NMOS W=2.5u L=250n M=2 AS=4.5p PS=13.6u AD=3.125p $\mathrm{PD}=7.5 \mathrm{u}$

MP1 1 In Vdd Vdd PMOS W=2.5u L=250n M=2 AS=4.5p PS=13.6u AD=3.125p

$\mathrm{PD}=7.5 \mathrm{u}$

MP2 Out 1 Vdd Vdd PMOS W=2.5u L=250n M=4 AS=7.625p PS=21.1u AD=6.25p

$\mathrm{PD}=15 \mathrm{u}$

.ends

.subckt BUF1N50 In Out Gnd Vdd

*------- Devices: SPICE.ORDER > 0

MNMOS_1 Out N_4 Gnd Gnd NMOS W=2.5u L=250n AS=2.25p PS=6.8u

$\mathrm{AD}=2.25 \mathrm{p} \mathrm{PD}=6.8 \mathrm{u}$

MNMOS_2 N_4 In Gnd Gnd NMOS W=2.5u L=250n AS=2.25p PS=6.8u AD=2.25p

$\mathrm{PD}=6.8 \mathrm{u}$

MPMOS_1 Out N_4 Vdd Vdd PMOS W=2.5u L=250n AS=2.25p PS=6.8u

$\mathrm{AD}=2.25 \mathrm{p} \mathrm{PD}=6.8 \mathrm{u}$ 
MPMOS_3 N_4 In Vdd Vdd PMOS W=2.5u L=250n AS=2.25p PS=6.8u AD $=2.25 p$

$\mathrm{PD}=6.8 \mathrm{u}$

.ends

.subckt DFFC ClB Clk Data Q QB Gnd Vdd

*------ Devices: SPICE.ORDER $<0$--------

* Design: LogicGates / Cell: DFFC / View: Main / Page:

* Designed by: Tanner EDA Library Development Team

* Organization: Tanner EDA - Tanner Research, Inc.

* Info: D Flip-Flop with Clear

* Date: 6/11/2007 3:39:34 PM

* Revision: 43

* Design: LogicGates / Cell: DFFC / View: Main / Page:

* Designed by: Tanner EDA Library Development Team

* Organization: Tanner EDA - Tanner Research, Inc.

* Info: D Flip-Flop with Clear

* Date: 6/11/2007 3:39:34 PM

* Revision: 43

*------- Devices: SPICE.ORDER > 0 --------

MN1 5 Data Gnd 0 NMOS W=1.9u L=250n AS=1.71p PS=5.6u AD=1.71p PD=5.6u

MN2 4 CB 50 NMOS W=1.9u L=250n AS=1.71p PS=5.6u AD=1.71p PD=5.6u 
MN3 71080 NMOS W=1.9u L=250n AS=1.71p PS=5.6u AD=1.71p PD=5.6u MN4 4 C 70 NMOS $W=1.9 u$ L=250n AS=1.71p PS=5.6u AD=1.71p PD=5.6u MN27 14 Q Gnd 0 NMOS W=2.5u L=250n AS=2.25p PS=6.8u AD=2.25p PD=6.8u MN11 CB Clk Gnd 0 NMOS W=1u L=250n AS=900f PS=3.8u AD=900f PD=3.8u MN12 C CB Gnd 0 NMOS W=1u L=250n AS=900f PS=3.8u AD=900f PD=3.8u MN13 8 ClB Gnd 0 NMOS W=1.9u L=250n AS=1.71p PS=5.6u AD=1.71p PD=5.6u MN14 104 Gnd 0 NMOS W=1.9u L=250n AS=1.71p PS=5.6u AD=1.71p PD=5.6u MN15 Q 12 Gnd 0 NMOS W=2.5u L=250n AS=2.25p PS=6.8u AD=2.25p PD=6.8u MN16 12 CB QB 0 NMOS W=1.9u L=250n AS=1.71p PS=5.6u AD=1.71p PD=5.6u MN17 QB ClB 140 NMOS W=2.5u L=250n AS=2.25p PS=6.8u AD=2.25p $\mathrm{PD}=6.8 \mathrm{u}$

MN18 12 C 13 0 NMOS W=1.9u L=250n AS=1.71p PS=5.6u AD=1.71p PD=5.6u MN19 1310 Gnd 0 NMOS W=1.9u L=250n AS=1.71p PS=5.6u AD=1.71p PD=5.6u MP1 4 C 3 Vdd PMOS W=2.15u L=250n AS=1.935p PS=6.1u AD=1.935p PD=6.1u MP2 3 Data Vdd Vdd PMOS W=2.15u L=250n AS=1.935p PS=6.1u AD=1.935p $\mathrm{PD}=6.1 \mathrm{u}$

MP3 4 CB 6 Vdd PMOS W=1.75u L=250n AS=1.575p PS=5.3u AD=1.575p $\mathrm{PD}=5.3 \mathrm{u}$

MP11 CB Clk Vdd Vdd PMOS W=1u L=250n AS=900f PS=3.8u AD=900f PD=3.8u MP4 610 Vdd Vdd PMOS W=1.75u L=250n AS=1.575p PS=5.3u AD=1.575p $P D=5.3 u$

MP12 C CB Vdd Vdd PMOS W=1u L=250n AS=900f PS=3.8u AD=900f $P D=3.8 u$ MP13 4 CB 9 Vdd PMOS W=1.4u L=250n AS=1.26p PS=4.6u AD=1.26p PD=4.6u 
MP14 104 Vdd Vdd PMOS W=1.9u L=250n AS=1.71p PS=5.6u AD=1.71p

$\mathrm{PD}=5.6 \mathrm{u}$

MP17 Q 12 Vdd Vdd PMOS W=3.4u L=250n AS=3.06p PS=8.6u AD=3.06p

$\mathrm{PD}=8.6 \mathrm{u}$

MP9 9 ClB Vdd Vdd PMOS W=1.5u L=250n AS=1.35p PS=4.8u AD=1.35p

$\mathrm{PD}=4.8 \mathrm{u}$

MP22 QB Q Vdd Vdd PMOS W=3u L=250n AS=2.7p PS=7.8u AD=2.7p PD=7.8u

MP26 12 CB 11 Vdd PMOS W=2u L=250n AS=1.8p PS=5.8u AD=1.8p PD=5.8u

MP27 1110 Vdd Vdd PMOS W=2u L=250n AS=1.8p PS=5.8u AD=1.8p PD=5.8u

MP28 12 C QB Vdd PMOS W=2u L=250n AS=1.8p PS=5.8u AD=1.8p PD=5.8u

MP29 QB ClB Vdd Vdd PMOS W=3u L=250n AS=2.7p PS=7.8u AD=2.7p PD=7.8u

.ends

.subckt Mux2 A B Out Sel Gnd Vdd

*-------- Devices: SPICE.ORDER < 0 --------

* Design: LogicGates / Cell: Mux2 / View: Main / Page:

* Designed by: Tanner EDA Library Development Team

* Organization: Tanner EDA - Tanner Research, Inc.

* Info: 2 Input Multiplexer

* Date: 5/21/2007 2:43:54 PM

* Revision: 37

*------- Devices: SPICE.ORDER > 0 
MN5 6 G Gnd 0 NMOS W=2.5u L=250n AS=2.25p PS=6.8u AD=2.25p PD=6.8u MN6 Out 3 Gnd 0 NMOS W=2.5u L=250n M=2 AS=4.5p PS=13.6u AD=3.125p $\mathrm{PD}=7.5 \mathrm{u}$

MN1 G Sel Gnd 0 NMOS W=2.5u L=250n AS=2.25p PS=6.8u AD=2.25p PD=6.8u MN2 3 A 40 NMOS $W=2.5 u$ L=250n AS=2.25p PS=6.8u AD=2.25p PD=6.8u MN3 4 Sel Gnd 0 NMOS W=2.5u L=250n AS=2.25p PS=6.8u AD=2.25p PD=6.8u

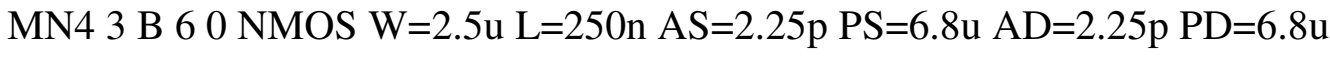
MP1 G Sel Vdd Vdd PMOS W=2.5u L=250n AS=2.25p PS=6.8u AD=2.25p $\mathrm{PD}=6.8 \mathrm{u}$

MP2 2 G Vdd Vdd PMOS W=2.5u L=250n AS=2.25p PS=6.8u AD=2.25p PD=6.8u MP3 3 A 2 Vdd PMOS W=2.5u L=250n AS=2.25p PS=6.8u AD=2.25p PD=6.8u MP4 5 Sel Vdd Vdd PMOS W=2.5u L=250n AS=2.25p PS=6.8u AD=2.25p PD=6.8u MP5 3 B 5 Vdd PMOS $W=2.5 u ~ L=250 n ~ A S=2.25 p$ PS $=6.8 u$ AD $=2.25 p$ PD $=6.8 u$ MP6 Out 3 Vdd Vdd PMOS W=2.5u L=250n M=2 AS=4.5p PS=13.6u AD=3.125p $\mathrm{PD}=7.5 \mathrm{u}$ .ends

.subckt BUF2 In Out Gnd Vdd XBuf1_1 In N_1 Gnd Vdd Buf1 XBuf1_2 N_1 Out Gnd Vdd Buf1 .ends .subckt BUF4 In Out Gnd Vdd 
XBuf1_1 In N_1 Gnd Vdd Buf1

XBuf1_2 N_1 N_2 Gnd Vdd Buf1

XBuf1_3 N_2 N_3 Gnd Vdd Buf1

XBuf1_4 N_3 Out Gnd Vdd Buf1

.ends

.subckt BUF8 In Out Gnd Vdd

XBuf1_1 In N_2 Gnd Vdd Buf1

XBuf1_2 N_2 N_3 Gnd Vdd Buf1

XBuf1_3 N_3 N_4 Gnd Vdd Buf1

XBuf1_4 N_4 N_1 Gnd Vdd Buf1

XBuf1_5 N_5 Out Gnd Vdd Buf1

XBuf1_6 N_6 N_5 Gnd Vdd Buf1

XBuf1_7 N_7 N_6 Gnd Vdd Buf1

XBuf1_8 N_1 N_7 Gnd Vdd Buf1

.ends

.subckt BUFD In Out Gnd Vdd

XBuf1_1 In N_6 Gnd Vdd Buf1

XBuf1_2 N_6 N_1 Gnd Vdd Buf1

XBuf1_3 N_2 N_3 Gnd Vdd Buf1

XBuf1_4 N_3 Out Gnd Vdd Buf1

XBuf1_5 N_1 N_4 Gnd Vdd Buf1 
XBuf1_6 N_4 N_2 Gnd Vdd Buf1

.ends

.subckt BUFM In Out Gnd Vdd

XBuf1_1 In N_1 Gnd Vdd Buf1

XBUF1N50_1 N_1 Out Gnd Vdd BUF1N50

.ends

********* Simulation Settings - Parameters and SPICE Options $* * * * * * * * *$

*-------- Devices: SPICE.ORDER == 0 --------

XMux2_1 N_18 N_22 N_6 Q2 Gnd Vdd Mux2

XMux2_2 N_17 N_23 N_3 Q1 Gnd Vdd Mux2

XMux2_3 N_20 N_21 N_13 Q3 Gnd Vdd Mux2

XBUF4_1 N_13 N_8 Gnd Vdd BUF4

XBUF8_1 X N_11 Gnd Vdd BUF8

XBUF4_2 N_10 N_19 Gnd Vdd BUF4

XBUF8_2 Y N_9 Gnd Vdd BUF8 
XBUFD_1 N_9 N_12 Gnd Vdd BUFD

XBUFD_2 N_19 N_14 Gnd Vdd BUFD

XBUFD_3 N_5 N_16 Gnd Vdd BUFD

XBUFD_4 X N_20 Gnd Vdd BUFD

XBUFD_5 N_11 N_21 Gnd Vdd BUFD

XBUFD_6 N_8 N_22 Gnd Vdd BUFD

XBUFD_7 N_13 N_18 Gnd Vdd BUFD

XBUFD_8 N_1 N_23 Gnd Vdd BUFD

XBUFD_9 N_6 N_17 Gnd Vdd BUFD

XBuf1_1 N_15 N_2 Gnd Vdd Buf1

XBUFM_1 N_12 N_10 Gnd Vdd BUFM

XBUFM_2 N_14 N_7 Gnd Vdd BUFM

XBUFM_3 N_16 N_15 Gnd Vdd BUFM

XBUF2_1 N_6 N_1 Gnd Vdd BUF2

XBUF2_2 N_7 N_5 Gnd Vdd BUF2

XDFFC_1 N_4 N_19 N_13 N_24 Q2 Gnd Vdd DFFC

XDFFC_2 N_4 N_5 N_6 N_25 Q1 Gnd Vdd DFFC

XDFFC_3 N_4 N_2 N_3 N_26 Q0 Gnd Vdd DFFC

XDFFC_4 N_4 N_9 X N_27 Q3 Gnd Vdd DFFC

*-------- Devices: SPICE.ORDER > 0 --------

VVdd Vdd Gnd DC 5

Vreset N_4 Gnd BIT(\{00111111111111111111111111111111\} PW=300p RT=1p

$\mathrm{FT}=1 \mathrm{p} \mathrm{LT}=300 \mathrm{p}$ HT=300p) 
VX X Gnd BIT(\{0000000011111111111111111111111 $\}$ PW=300p RT=1p FT=1p

$\mathrm{LT}=300 \mathrm{p} \mathrm{HT}=300 \mathrm{p})$

VY Y Gnd BIT( $\{000011111111111111111111111\} \quad P W=300 p$ RT $=1 p \quad F T=1 p$

LT=300p HT=300p)

********* Simulation Settings - Analysis section $* * * * * * * * *$

.tran $2 \mathrm{p} 7 \mathrm{n}$ start $=0$

.print TRAN v(X) v(Y) v(Q3) v(Q2) v(Q1) v(Q0)

********* Simulation Settings - Additional SPICE commands $* * * * * * * * * *$

.end 